\title{
Geometric realization of Dynkin quiver type quantum affine Schur-Weyl duality
}

\author{
Ryo Fujita*
}

December 2, 2019

\begin{abstract}
For a Dynkin quiver $Q$ of type ADE and a sum $\beta$ of simple roots, we construct a bimodule over the quantum loop algebra and the quiver Hecke algebra of the corresponding type via equivariant $K$-theory, imitating Ginzburg-Reshetikhin-Vasserot's geometric realization of the quantum affine Schur-Weyl duality. Our construction is based on HernandezLeclerc's isomorphism between a certain graded quiver variety and the space of representations of the quiver $Q$ of dimension vector $\beta$. We identify the functor induced from our bimodule with Kang-Kashiwara-Kim's generalized quantum affine Schur-Weyl duality functor. As a by-product, we verify a conjecture by Kang-Kashiwara-Kim on the simpleness of some poles of normalized $R$-matrices for any quiver $Q$ of type ADE.
\end{abstract}

\section{Introduction}

For a fixed pair $(n, d)$ of positive integers, we have the following two fundamental objects: the complex simple Lie algebra $\mathfrak{s l}_{n+1}$ of type $\mathrm{A}_{n}$ and the symmetric group $\mathfrak{S}_{d}$ of degree $d$. The natural $\left(\mathfrak{s l}_{n+1}, \mathfrak{S}_{d}\right)$-bimodule structure on the tensor power $\left(\mathbb{C}^{n+1}\right)^{\otimes d}$ produces a close relationship between their representation theories. This phenomenon is known as the classical Schur-Weyl duality and has many interesting variants.

The quantum affine Schur-Weyl duality is a variant involving their quantum affinizations: the quantum loop algebra $U_{q}\left(L \mathfrak{s l}_{n+1}\right)$ of $\mathfrak{s l}_{n+1}$ and the affine Hecke algebra $H_{d}^{\text {af }}(q)$ of $G L_{d}$. Both algebras are defined over $\mathbb{k}:=\mathbb{Q}(q)$. Here we equip the tensor power $\mathbb{V}^{\otimes d}$ of the natural representation $\mathbb{V}:=\mathbb{k}^{n+1}\left[z^{ \pm 1}\right]$ of $U_{q}\left(L \mathfrak{s l}_{n+1}\right)$ with a commuting right action of $H_{d}^{\text {af }}\left(q^{2}\right)$ using the $R$-matrices. Chari-Pressley [1] proved that the induced functor

$$
H_{d}^{\mathrm{af}}\left(q^{2}\right)-\bmod \rightarrow U_{q}\left(L \mathfrak{s l}_{n+1}\right)-\bmod ; \quad M \mapsto \mathbb{V}^{\otimes d} \otimes_{H_{d}^{\mathrm{af}}\left(q^{2}\right)} M
$$

* Department of Mathematics, Kyoto University, Oiwake Kita-Shirakawa Sakyo Kyoto 6068502 JAPAN, E-mail:rfujita@math.kyoto-u.ac.jp 
gives an equivalence between suitable subcategories of finite-dimensional modules.

The quantum affine Schur-Weyl duality has a beautiful geometric realization due to Ginzburg-Reshetikhin-Vasserot [6]. Here we recall their construction briefly. Let $\mu_{d}: \mathcal{F}_{d} \rightarrow \mathcal{N}_{d}$ be the Springer resolution of the nilpotent cone $\mathcal{N}_{d}$ of $\mathfrak{g l}_{d}(\mathbb{C})$, where $\mathcal{F}_{d}$ is the cotangent bundle of the full flag variety of $G L_{d}(\mathbb{C})$. The morphism $\mu_{d}$ is equivariant with respect to a natural action of the group $\mathbb{G}_{d}:=G L_{d}(\mathbb{C}) \times \mathbb{C}^{\times}$, where $\mathbb{C}^{\times}$acts as the scalar multiplication on the cone $\mathcal{N}_{d}$. Due to Ginzburg and Kazhdan-Lusztig, the affine Hecke algebra $H_{d}^{\text {af }}\left(q^{2}\right)$ is isomorphic to the convolution algebra $K^{\mathbb{G}_{d}}\left(\mathcal{Z}_{d}\right) \otimes_{A} \mathbb{k}$ of the equivariant $K$-group of the Steinberg variety $\mathcal{Z}_{d}:=\mathcal{F}_{d} \times_{\mathcal{N}_{d}} \mathcal{F}_{d}$, where $A=R\left(\mathbb{C}^{\times}\right)=\mathbb{Z}\left[v^{ \pm 1}\right]$ is the representation ring of $\mathbb{C}^{\times}$and $-\otimes_{A} \mathbb{k}$ means the specialization $v \mapsto q$. On the other hand, we consider another Steinberg type variety $Z_{d}:=\mathfrak{M}_{d} \times_{\mathcal{N}_{d}} \mathfrak{M}_{d}$. Here $\mathfrak{M}_{d}$ is the cotangent bundle of the variety of partial flags in $\mathbb{C}^{d}$ of length $\leq n+1$. Due to Ginzburg-Vasserot, there is an algebra homomorphism $\Phi: U_{q}\left(L \mathfrak{s l}_{n+1}\right) \rightarrow$ $K^{\mathbb{G}_{d}}\left(Z_{d}\right) \otimes_{A} \mathbb{k}$ with some good properties. Based on these facts, GinzburgReshetikhin-Vasserot considered the intermediary fiber product $\mathfrak{M}_{d} \times_{\mathcal{N}_{d}} \mathcal{F}_{d}$ and identified its equivariant $K$-group with the bimodule $\mathbb{V}^{\otimes d}$. More precisely, they established an isomorphism $\mathbb{V}^{\otimes d} \cong K^{\mathbb{G}_{d}}\left(\mathfrak{M}_{d} \times_{\mathcal{N}_{d}} \mathcal{F}_{d}\right) \otimes_{A} \mathbb{k}$ making the following diagram commute:

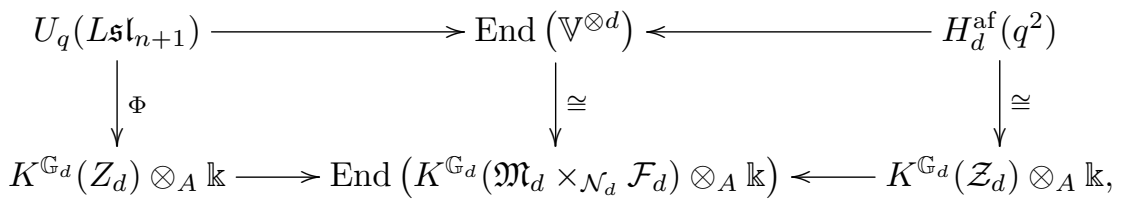

where horizontal arrows denote the bimodule structures.

Recently, in a series of papers [9, 10, 11, 12], Kang, Kashiwara, Kim and Oh established some interesting generalized versions of the quantum affine SchurWeyl duality. One of them (treated in [10] by Kang-Kashiwara-Kim) is associated with a pair $(Q, \beta)$ of a Dynkin quiver $Q$ of type ADE and a sum $\beta=\sum_{i} d_{i} \alpha_{i}$ of simple roots, which plays a similar role as the pair $(n, d)$ in the previous paragraphs. One player is the quantum loop algebra $U_{q}(L \mathfrak{g})$ of the complex simple Lie algebra $\mathfrak{g}$ whose Dynkin diagram is the underlying graph of $Q$. The other is the quiver Hecke (KLR) algebra $H_{Q}(\beta)$ associated with $(Q, \beta)$, or actually its completion $\widehat{H}_{Q}(\beta)$ along the grading. The quiver Hecke algebra $H_{Q}(\beta)$ is regarded as a generalization of the affine Hecke algebra $H_{d}^{\text {af }}(q)$ from the viewpoint of the categorification of the quantum group. Inspired by the work of Hernandez-Leclerc [8], Kang-Kashiwara-Kim [10] constructed on a left $U_{q}(L \mathfrak{g})$-module $\widehat{V}^{\otimes \beta}$ which is a direct sum of some tensor products of affinized fundamental modules a commuting right action of the algebra $\widehat{H}_{Q}(\beta)$ by using the normalized $R$-matrices. However, to make the $\widehat{H}_{Q}(\beta)$-action well-defined, we need to assume the simpleness of some poles of the normalized $R$-matrices. This assumption was verified for type AD in [10] by an explicit computation of the denominators of the normalized $R$-matrices. On the other hand, for type 
$\mathrm{E}$, this remains a conjecture. Under this well-definedness assumption, KangKashiwara-Kim [10] also proved that the induced functor

$$
\widehat{H}_{Q}(\beta)-\bmod _{\mathrm{fd}} \rightarrow U_{q}(L \mathfrak{g})-\bmod _{\mathrm{fd}} ; \quad M \mapsto \widehat{V}^{\otimes \beta} \otimes_{\widehat{H}_{Q}(\beta)} M
$$

is exact, factors through the $\beta$-block $\mathcal{C}_{Q, \beta}$ of a monoidal full subcategory $\mathcal{C}_{Q}$ of $U_{q}(L \mathfrak{g})-\bmod _{\mathrm{fd}}$ introduced by Hernandez-Leclerc [8] and gives a bijection between the simple isomorphism classes. More recently, the author [5] proved that it actually gives an equivalence $\widehat{H}_{Q}(\beta)-\bmod _{\mathrm{fd}} \simeq \mathcal{C}_{Q, \beta}$ by using the notion of affine highest weight category. Note that here we forget the gradings by working with the completion $\widehat{H}_{Q}(\beta)$.

In the present paper, we give a geometric realization of the bimodule $\widehat{V}^{\otimes \beta}$ imitating Ginzburg-Reshetikhin-Vasserot's realization. In our case, the nilpotent cone $\mathcal{N}_{d}$ is replaced by the space $E_{\beta}$ of representations of the quiver $Q$ over $\mathbb{C}$ of dimension vector $\beta$. The group $G_{\beta}:=\prod_{i} G L_{d_{i}}(\mathbb{C})$ naturally acts on $E_{\beta}$. Instead of the Springer resolution $\mathcal{F}_{d} \rightarrow \mathcal{N}_{d}$, we consider a proper morphism $\mathcal{F}_{\beta} \rightarrow E_{\beta}$ from a "quiver flag variety" $\mathcal{F}_{\beta}$ introduced by Lusztig in order to construct the canonical basis of the quantum group. Varagnolo-Vasserot [21] proved that the quiver Hecke algebra $H_{Q}(\beta)$ is isomorphic to the convolution algebra of the equivariant Borel-Moore homology $H_{*}^{G_{\beta}}\left(\mathcal{Z}_{\beta}, \mathbb{k}\right)$, where $\mathcal{Z}_{\beta}:=$ $\mathcal{F}_{\beta} \times_{E_{\beta}} \mathcal{F}_{\beta}$. After completion, it is isomorphic to the completed equivariant $K$ group $\widehat{K}^{G_{\beta}}\left(\mathcal{Z}_{\beta}\right)_{\mathbb{k}}$. On the $U_{q}(L \mathfrak{g})$-side, we consider a canonical $G_{\beta}$-equivariant proper morphism $\mathfrak{M}_{\beta}^{\bullet} \rightarrow \mathfrak{M}_{0, \beta}^{\bullet}$ between certain graded quiver varieties. By Nakajima [17], we have an algebra homomorphism $\widehat{\Phi}_{\beta}: U_{q}(L \mathfrak{g}) \rightarrow \widehat{K}^{G_{\beta}}\left(Z_{\beta}^{\bullet}\right)_{\mathbb{k}}$, where $Z_{\beta}^{\bullet}:=\mathfrak{M}_{\beta}^{\bullet} \times_{\mathfrak{M}_{0, \beta}} \mathfrak{M}_{\beta}^{\bullet}$. The key of our construction is a $G_{\beta}$-equivariant isomorphism $\mathfrak{M}_{0, \beta}^{\bullet} \cong E_{\beta}$ due to Hernandez-Leclerc [8], which was originally established in order to give a geometric interpretation to their isomorphism between the Grothendieck ring $K\left(\mathcal{C}_{Q}\right)$ and the coordinate ring of the maximal unipotent subgroup (see Remark 3.13). This allows us to form the intermediary fiber product $\mathfrak{M}_{\beta}^{\bullet} \times_{E_{\beta}} \mathcal{F}_{\beta}$.

Theorem 1.1 (=Theorem $4.4+4.6$, see also Remark 4.8). There is an isomorphism

$$
\widehat{V}^{\otimes \beta} \cong \widehat{K}^{G_{\beta}}\left(\mathfrak{M}_{\beta}^{\bullet} \times_{E_{\beta}} \mathcal{F}_{\beta}\right)_{\mathbb{k}}
$$

such that the following diagram commutes (up to a twist):

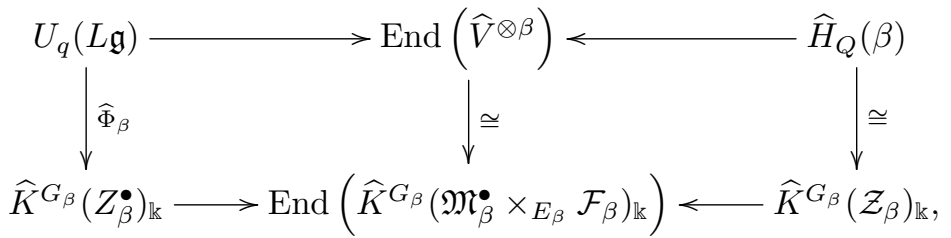

where the horizontal arrows denote the bimodule structures.

Actually, our geometric construction of the $\widehat{H}_{Q}(\beta)$-action is independent of that of [10], which shares the same characterization of the actions. Therefore, their comparison yields a uniform proof of: 
Theorem 1.2 (=Corollary 4.7). Kang-Kashiwara-Kim's conjecture [10, Conjecture 4.3.2] on the simpleness of some specific poles of normalized $R$-matrices for tensor products of fundamental modules is true for any quiver $Q$ of type ADE.

Besides, a discussion involving geometric extension algebras yields another proof of the equivalence $\widehat{H}_{Q}(\beta)-\bmod _{\mathrm{fd}} \simeq \mathcal{C}_{Q, \beta}$ given by the bimodule $\widehat{V}^{\otimes \beta}$ without using affine highest weight categories (Theorem 4.9). We would also remark that we do not use any results of [9], [10] for our proofs.

The present paper is organized as follows. In Section 2, we recall the definition of graded quiver varieties $\mathfrak{M}_{\beta}^{\bullet}$ and $\mathfrak{M}_{0, \beta}^{\bullet}$, and Hernandez-Leclerc's isomorphism $\mathfrak{M}_{0, \beta}^{\bullet} \cong E_{\beta}$. In Section 3, we study the convolution algebra $\widehat{K}^{G_{\beta}}\left(\mathcal{Z}_{\beta}\right)_{\mathbb{k}}$ (resp. $\widehat{K}^{G_{\beta}}\left(Z_{\beta}^{\bullet}\right)_{\mathbb{k}}$ ) and recall its relation to the quiver Hecke algebra $H_{Q}(\beta)$ (resp. the quantum loop algebra $\left.U_{q}(L \mathfrak{g})\right)$. In the final section 4, we study the structure of the bimodule $\widehat{K}^{G_{\beta}}\left(\mathfrak{M}_{\beta}^{\bullet} \times_{E_{\beta}} \mathcal{F}_{\beta}\right)_{\mathbb{k}}$.

While the author was writing this paper, there appeared a preprint by OhScrimshaw [19] in arXiv which also proves Theorem 1.2 by a different approach. They compute the denominators of the normalized $R$-matrices for type $\mathrm{E}$ explicitly with a computer.

Acknowledgment. The author thanks Syu Kato for helpful discussions and comments. He also thanks Ryosuke Kodera for suggesting him to study the geometric realization of the generalized quantum affine Schur-Weyl duality.

Convention. An algebra $A$ is associative and unital. We denote by $A^{\text {op }}$ (resp. $A^{\times}$) the opposite algebra (resp. the set of invertible elements) of $A$ and by $A$-mod the category of left $A$-modules. Working over a base field $\mathbb{F}$, the symbol $\otimes$ (resp. Hom) stands for $\otimes_{\mathbb{F}}($ resp. Hom $\mathbb{F}$ ) if there is no other clarification. If $A$ is an $\mathbb{F}$-algebra, we denote by $A$ - $\bmod _{\mathrm{fd}}$ the category of finite-dimensional left $A$-modules.

\section{Hernandez-Leclerc's isomorphism}

\subsection{Notation}

Throughout this paper, we fix a finite-dimensional complex simple Lie algebra $\mathfrak{g}$ of type ADE and a quiver $Q=(I, \Omega)$ whose underlying graph is the Dynkin diagram of $\mathfrak{g}$, where $I=\{1,2, \ldots, n\}$ (resp. $\Omega$ ) is the set of vertices (resp. arrows). For an arrow $h \in \Omega$, let $h^{\prime}, h^{\prime \prime} \in I$ denote its origin and goal respectively. We write $i \sim j$ (resp. $i \rightarrow j$ ) if there is an arrow $h \in \Omega$ such that $\{i, j\}=\left\{h^{\prime}, h^{\prime \prime}\right\}$ (resp. $\left.(i, j)=\left(h^{\prime}, h^{\prime \prime}\right)\right)$. Then the Cartan matrix $\left(a_{i j}\right)_{i, j \in I}$ of $\mathfrak{g}$ is given by

$$
a_{i j}= \begin{cases}2 & \text { if } i=j \\ -1 & \text { if } i \sim j \\ 0 & \text { otherwise }\end{cases}
$$


Let $\mathrm{P}^{\vee}=\bigoplus_{i \in I} \mathbb{Z} h_{i}$ be the coroot lattice of $\mathfrak{g}$. The fundamental weights $\left\{\varpi_{i}\right\}_{i \in I}$ form a basis of the weight lattice $\mathrm{P}=\operatorname{Hom}_{\mathbb{Z}}\left(\mathrm{P}^{\vee}, \mathbb{Z}\right)$ which is dual to $\left\{h_{i}\right\}_{i \in I}$. Let $\alpha_{i}=\sum_{j \in I} a_{i j} \varpi_{j}$ be the $i$-th simple root and $\mathrm{Q}=\bigoplus_{i \in I} \mathbb{Z} \alpha_{i} \subset \mathrm{P}$ be the root lattice. We put $\mathrm{P}^{+}=\sum_{i \in I} \mathbb{Z}_{\geq 0} \varpi_{i}$ and $\mathrm{Q}^{+}=\sum_{i \in I} \mathbb{Z}_{\geq 0} \alpha_{i}$. The Weyl group is the finite group $W$ of linear transformations on $\mathrm{P}$ generated by the set $\left\{r_{i}\right\}_{i \in I}$ of simple reflections, which are given by $r_{i}(\lambda):=\lambda-\lambda\left(h_{i}\right) \alpha_{i}$ for $\lambda \in \mathrm{P}$. The set $\mathrm{R}^{+}$of positive roots is defined by $\mathrm{R}^{+}=\left(W\left\{\alpha_{i}\right\}_{i \in I}\right) \cap \mathrm{Q}^{+}$.

\subsection{Representations of Dynkin quiver}

For an element $\beta \in \mathrm{Q}^{+}$, we fix an $I$-graded $\mathbb{C}$-vector space $D=\bigoplus_{i \in I} D_{i}$ such that $\underline{\operatorname{dim}} D:=\sum_{i \in I}\left(\operatorname{dim} D_{i}\right) \alpha_{i}=\beta$. Let us consider the space

$$
E_{\beta}:=\bigoplus_{h \in \Omega} \operatorname{Hom}\left(D_{h^{\prime}}, D_{h^{\prime \prime}}\right)
$$

of representations of the quiver $Q$ of dimension vector $\beta$. On the space $E_{\beta}$, the group $G_{\beta}:=\prod_{i \in I} G L\left(D_{i}\right)$ acts by conjugation. The set $G_{\beta} \backslash E_{\beta}$ of $G_{\beta}$-orbits is naturally in bijection with the set of isomorphism classes of representations of the quiver $Q$ of dimension vector $\beta$. By Gabriel's theorem, for each $\alpha \in \mathrm{R}^{+}$there exists an indecomposable representation $M_{\alpha}$ such that $\underline{\operatorname{dim}} M_{\alpha}=\alpha$ uniquely up to isomorphism. The correspondence $\alpha \mapsto M_{\alpha}$ gives a bijection between the set $\mathrm{R}^{+}$of positive roots and the set of isomorphism classes of indecomposable objects of the category $\operatorname{Rep} Q$ of finite-dimensional representations of $Q$. Hence, the set

$$
\operatorname{KP}(\beta):=\left\{\left(m_{\alpha}\right) \in\left(\mathbb{Z}_{\geq 0}\right)^{\mathrm{R}^{+}} \mid \sum_{\alpha \in \mathrm{R}^{+}} m_{\alpha} \alpha=\beta\right\}
$$

of Kostant partitions of $\beta$ labels the set of $G_{\beta}$-orbits: $G_{\beta} \backslash E_{\beta}=\left\{\mathbb{O}_{\mathbf{m}}\right\}_{\mathbf{m} \in \operatorname{KP}(\beta)}$, where for each $\mathbf{m}=\left(m_{\alpha}\right) \in \operatorname{KP}(\beta)$, the $G_{\beta}$-orbit $\mathbb{O}_{\mathbf{m}}$ corresponds to the isomorphism class of the representation $\bigoplus_{\alpha \in \mathrm{R}^{+}} M_{\alpha}^{\oplus m_{\alpha}}$. We have the natural $G_{\beta^{-}}$orbit stratification

$$
E_{\beta}=\bigsqcup_{\mathbf{m} \in \mathrm{KP}(\beta)} \mathbb{O}_{\mathbf{m}}
$$

\subsection{Repetition quiver}

We fix a height function $\xi: I \rightarrow \mathbb{Z} ; i \mapsto \xi_{i}$ of the quiver $Q$ i.e. it satisfies $\xi_{i}=\xi_{j}+1$ if $i \rightarrow j$. Such a function $\xi$ is determined up to adding a constant. Choose a total ordering $I=\left\{i_{1}, i_{2}, \ldots, i_{n}\right\}$ such that $\xi_{i_{1}} \geq \xi_{i_{2}} \geq \cdots \geq \xi_{i_{n}}$ and define the corresponding Coxeter element $c:=r_{i_{1}} r_{i_{2}} \cdots r_{i_{n}} \in W$.

The repetition quiver $\widehat{Q}=(\widehat{I}, \widehat{\Omega})$ is an infinite quiver defined by

$$
\begin{aligned}
\widehat{I} & :=\left\{(i, p) \in I \times \mathbb{Z} \mid p-\xi_{i} \in 2 \mathbb{Z}\right\}, \\
\widehat{\Omega} & :=\{(i, p) \rightarrow(j, p+1) \mid(i, p),(j, p+1) \in \widehat{I}, i \sim j\} .
\end{aligned}
$$

It is well-known (cf. [7]) that there exists an isomorphism $\phi$ from the AuslanderReiten quiver of the derived category $D^{b}(\operatorname{Rep} Q)$ to the repetition quiver $\widehat{Q}$, 
which depends on the choice of $\xi$ and is described as follows. Since each indecomposable object of $D^{b}(\operatorname{Rep} Q)$ is isomorphic to a unique stalk complex $M_{\alpha}[k]$ for some $(\alpha, k) \in \mathrm{R}^{+} \times \mathbb{Z}$, we have a bijection between the sets of vertices

$$
\mathrm{R}^{+} \times \mathbb{Z} \ni(\alpha, k) \mapsto \phi\left(M_{\alpha}[k]\right) \in \widehat{I},
$$

which we denote by the same symbol $\phi$. This bijection $\phi: \mathrm{R}^{+} \times \mathbb{Z} \rightarrow \widehat{I}$ is determined inductively as follows:

- For each $i \in I$, we put $\gamma_{i}:=\sum_{j} \alpha_{j}$ where $j$ runs all the vertices $j \in I$ such that there is a path in $Q$ from $j$ to $i$. Then $M_{\gamma_{i}}$ is an injective hull of the 1-dimensional representation $M_{\alpha_{i}}$. We define $\phi\left(\gamma_{i}, 0\right):=\left(i, \xi_{i}\right)$;

- Inductively, if $\phi(\alpha, k)=(i, p)$ for $(\alpha, k) \in \mathrm{R}^{+} \times \mathbb{Z}$, then we define as:

$$
\begin{array}{rr}
\phi\left(c^{ \pm 1}(\alpha), k\right):=(i, p \mp 2) & \text { if } c^{ \pm 1}(\alpha) \in \mathrm{R}^{+}, \\
\phi\left(-c^{ \pm 1}(\alpha), k \mp 1\right):=(i, p \mp 2) & \text { if } c^{ \pm 1}(\alpha) \in-\mathrm{R}^{+} .
\end{array}
$$

In the followings, we only consider the restriction of the bijection $\phi$ on $\mathrm{R}^{+}=$ $\mathrm{R}^{+} \times\{0\}$, which we denote by the same symbol, i.e. we define $\phi(\alpha):=\phi(\alpha, 0)$ for $\alpha \in \mathrm{R}^{+}$.

\subsection{Graded quiver varieties}

In this subsection, we recall the definition of the graded quiver varieties. A basic reference is [17].

For elements $\nu=\sum_{i \in I} n_{i} \alpha_{i} \in \mathrm{Q}^{+}$and $\lambda=\sum_{i \in I} l_{i} \varpi_{i} \in \mathrm{P}^{+}$, we fix $I$-graded $\mathbb{C}$-vector spaces $V=\bigoplus_{i \in I} V_{i}, W=\bigoplus_{i \in I} W_{i}$ such that $\operatorname{dim} V_{i}=n_{i}, \operatorname{dim} W_{i}=l_{i}$ for each $i \in I$. We form the following space of linear maps:

$$
\mathbf{M}(\nu, \lambda):=\left(\bigoplus_{i \sim j} \operatorname{Hom}\left(V_{j}, V_{i}\right)\right) \oplus\left(\bigoplus_{i \in I} \operatorname{Hom}\left(W_{i}, V_{i}\right)\right) \oplus\left(\bigoplus_{i \in I} \operatorname{Hom}\left(V_{i}, W_{i}\right)\right)
$$

On the $\mathbb{C}$-vector space $\mathbf{M}(\nu, \lambda)$, the groups $G(\nu):=\prod_{i \in I} G L\left(V_{i}\right), G(\lambda):=$ $\prod_{i \in I} G L\left(W_{i}\right)$ act by conjugation and the 1 -dimensional torus $\mathbb{C}^{\times}$acts by the scalar multiplication. We write an element of $\mathbf{M}(\nu, \lambda)$ as a triple $(B, a, b)$ of linear maps $B=\bigoplus B_{i j}, a=\bigoplus a_{i}$ and $b=\bigoplus b_{i}$. Let $\mu=\bigoplus_{i \in I} \mu_{i}: \mathbf{M}(\nu, \lambda) \rightarrow$ $\bigoplus_{i \in I} \mathfrak{g l}\left(V_{i}\right)$ be the map given by

$$
\mu_{i}(B, a, b)=a_{i} b_{i}+\sum_{j \sim i} \varepsilon(i, j) B_{i j} B_{j i}
$$

where $\varepsilon(i, j):=1$ (resp. -1$)$ if $j \rightarrow i$ (resp. $i \rightarrow j$ ). A point $(B, a, b) \in \mu^{-1}(0)$ is said to be stable if there exists no non-zero $I$-graded subspace $V^{\prime} \subset V$ such that $B\left(V^{\prime}\right) \subset V^{\prime}$ and $V^{\prime} \subset \operatorname{Ker} b$. Let $\mu^{-1}(0)^{\text {st }}$ be the set of stable points, on which $G(\nu)$ acts freely. Then we consider a set-theoretic quotient

$$
\mathfrak{M}(\nu, \lambda):=\mu^{-1}(0)^{\mathrm{st}} / G(\nu) .
$$


It is known that this quotient has a structure of a non-singular quasi-projective variety which is isomorphic to a quotient in the geometric invariant theory. We also consider the affine algebro-geometric quotient

$$
\mathfrak{M}_{0}(\nu, \lambda):=\mu^{-1}(0) / / G(\nu)=\operatorname{Spec} \mathbb{C}\left[\mu^{-1}(0)\right]^{G(\nu)},
$$

together with a canonical projective morphism $\mathfrak{M}(\nu, \lambda) \rightarrow \mathfrak{M}_{0}(\nu, \lambda)$. These quotients $\mathfrak{M}(\nu, \lambda), \mathfrak{M}_{0}(\nu, \lambda)$ naturally inherit the actions of the group $\mathbb{G}(\lambda):=$ $G(\lambda) \times \mathbb{C}^{\times}$, which makes the canonical projective morphism into a $\mathbb{G}(\lambda)$-equivariant morphism.

For $\nu, \nu^{\prime} \in \mathrm{Q}^{+}$such that $\nu^{\prime}-\nu \in \mathrm{Q}^{+}$, there is a natural closed embedding $\mathfrak{M}_{0}(\nu, \lambda) \hookrightarrow \mathfrak{M}_{0}\left(\nu^{\prime}, \lambda\right)$. With respect to these embeddings, the family $\left\{\mathfrak{M}_{0}(\nu, \lambda)\right\}_{\nu \in Q^{+}}$forms an inductive system, which stabilizes at some $\nu \in \mathrm{Q}^{+}$. We consider the union (inductive limit) and obtain the following combined $\mathbb{G}(\lambda)$ equivariant morphism:

$$
\pi: \mathfrak{M}(\lambda):=\bigsqcup_{\nu} \mathfrak{M}(\nu, \lambda) \rightarrow \mathfrak{M}_{0}(\lambda):=\bigcup_{\nu} \mathfrak{M}_{0}(\nu, \lambda)
$$

We denote the fiber $\pi^{-1}(0)$ of the origin $0 \in \mathfrak{M}_{0}(\lambda)$ by $\mathfrak{L}(\lambda)=\bigsqcup_{\nu \in Q^{+}} \mathfrak{L}(\nu, \lambda)$. Note that $\mathfrak{M}(0, \lambda)=\mathfrak{L}(0, \lambda)$ consists of a single point.

Next we consider a free abelian monoid $\mathscr{P}^{+}=\mathbb{Z}_{\geq 0} \widehat{I}$ with the free generating set $\widehat{I}$. Define a homomorphism $\mathrm{cl}: \mathscr{P}^{+} \rightarrow \mathrm{P}^{+}$by $\mathrm{cl}(i, p)=\varpi_{i}$. For an element $\boldsymbol{\lambda}=\sum l_{i, p}(i, p) \in \mathscr{P}^{+}$with $\mathrm{cl}(\boldsymbol{\lambda})=\lambda$, we fix a decomposition $W_{i}=\bigoplus_{p} W_{i, p}$ such that $\operatorname{dim} W_{i, p}=l_{i, p}$ for each $(i, p) \in \widehat{I}$. Define a group homomorphism $f_{i}: \mathbb{C}^{\times} \rightarrow \prod_{p} G L\left(W_{i, p}\right) \subset G L\left(W_{i}\right)$ by $\left.f_{i}(t)\right|_{W_{i, p}}:=t^{p} \cdot \operatorname{id}_{W_{i, p}}$ for $t \in \mathbb{C}^{\times}$. We put $T(\boldsymbol{\lambda}):=\left(\prod_{i \in I} f_{i} \times \mathrm{id}\right)\left(\mathbb{C}^{\times}\right) \subset \mathbb{G}(\lambda)$ and consider the subvarieties of $T(\boldsymbol{\lambda})$-fixed points:

$$
\pi^{\bullet}:=\pi^{T(\boldsymbol{\lambda})}: \mathfrak{M}^{\bullet}(\boldsymbol{\lambda}):=\mathfrak{M}(\lambda)^{T(\boldsymbol{\lambda})} \rightarrow \mathfrak{M}_{0}^{\bullet}(\boldsymbol{\lambda}):=\mathfrak{M}_{0}(\lambda)^{T(\boldsymbol{\lambda})}
$$

We refer these varieties as the graded quiver varieties. We put $\mathfrak{L}^{\bullet}(\boldsymbol{\lambda}):=$ $\mathfrak{L}(\lambda)^{T(\boldsymbol{\lambda})}=\left(\pi^{\bullet}\right)^{-1}(0)$.

The centralizer of $T(\boldsymbol{\lambda})$ inside $\mathbb{G}(\lambda)$ is

$$
\mathbb{G}(\boldsymbol{\lambda}) \equiv G(\boldsymbol{\lambda}) \times \mathbb{C}^{\times}:=\prod_{(i, p) \in \widehat{I}} G L\left(W_{i, p}\right) \times \mathbb{C}^{\times} \subset \mathbb{G}(\lambda)
$$

which naturally acts on the varieties $\mathfrak{M}^{\bullet}(\boldsymbol{\lambda}), \mathfrak{M}_{0}^{\bullet}(\boldsymbol{\lambda}), \mathfrak{L} \bullet(\boldsymbol{\lambda})$. The morphism $\pi^{\bullet}$ is $\mathbb{G}(\boldsymbol{\lambda})$-equivariant.

\subsection{Hernandez-Leclerc's isomorphism}

Let $\mathscr{P}_{0}^{+} \subset \mathscr{P}^{+}$be the submonoid generated by the subset $\phi\left(\mathrm{R}^{+}\right) \subset \widehat{I}$. For an element $\beta:=\sum_{i \in I} d_{i} \alpha_{i} \in \mathrm{Q}^{+}$, we define $\boldsymbol{\lambda}_{\beta}:=\sum_{i \in I} d_{i} \phi\left(\alpha_{i}\right) \in \mathscr{P}_{0}^{+}$. In this case, we write $\pi_{\beta}: \mathfrak{M}_{\beta}^{\bullet} \rightarrow \mathfrak{M}_{0, \beta}^{\bullet}$ instead of $\pi^{\bullet}: \mathfrak{M}^{\bullet}\left(\boldsymbol{\lambda}_{\beta}\right) \rightarrow \mathfrak{M}_{0}^{\bullet}\left(\boldsymbol{\lambda}_{\beta}\right)$ for simplicity. For each $i \in I$, we identify the vector space $D_{i}$ in Subsection 2.2 
with the vector space $W_{\phi\left(\alpha_{i}\right)}$ in Subsection 2.4. This induces the identification $G_{\beta}=G\left(\boldsymbol{\lambda}_{\beta}\right)$. We write $\mathbb{G}_{\beta}, T_{\beta}$ instead of $\mathbb{G}\left(\boldsymbol{\lambda}_{\beta}\right), T\left(\boldsymbol{\lambda}_{\beta}\right)$ respectively. By the inclusion $G_{\beta}=G_{\beta} \times\{1\} \subset G_{\beta} \times \mathbb{C}^{\times}=\mathbb{G}_{\beta}$, the group $G_{\beta}$ is regarded as a subgroup of the group $\mathbb{G}_{\beta}$. Then the multiplication map $G_{\beta} \times T_{\beta} \rightarrow \mathbb{G}_{\beta}$ gives an isomorphism of algebraic groups

$$
G_{\beta} \times T_{\beta} \cong \mathbb{G}_{\beta} .
$$

We equip an action of the group $\mathbb{G}_{\beta}$ on the space $E_{\beta}$ via the projection $\mathbb{G}_{\beta} \cong$ $G_{\beta} \times T_{\beta} \rightarrow G_{\beta}$.

Theorem 2.1 (Hernandez-Leclerc [8] Theorem 9.11). There exists a $\mathbb{G}_{\beta}$-equivariant isomorphism of varieties

$$
\mathfrak{M}_{0, \beta}^{\bullet} \stackrel{\cong}{\rightrightarrows} E_{\beta} .
$$

Henceforth, we identify the graded quiver variety $\mathfrak{M}_{0, \beta}^{\bullet}$ with the space $E_{\beta}$ under the isomorphism in Theorem 2.1.

We recall some properties of fibers of the $\mathbb{G}_{\beta}$-equivariant morphism $\pi_{\beta}$ : $\mathfrak{M}_{\beta}^{\bullet} \rightarrow E_{\beta}$. By the injective map

$$
\mathrm{KP}(\beta) \ni\left(m_{\alpha}\right) \mapsto \sum_{\alpha} m_{\alpha} \phi(\alpha) \in \mathscr{P}_{0}^{+},
$$

we regard $\operatorname{KP}(\beta)$ as a subset of $\mathscr{P}_{0}^{+}$. Then we have a disjoint union decomposition

$$
\mathscr{P}_{0}^{+}=\bigsqcup_{\beta \in Q^{+}} \operatorname{KP}(\beta) .
$$

Proposition 2.2 (cf. [5] Section 3). Let $\mathbf{m} \in \mathrm{KP}(\beta)$ and pick a point $x_{\mathbf{m}} \in \mathbb{O}_{\mathbf{m}}$.

(1) We have an isomorphism $\pi_{\beta}^{-1}\left(x_{\mathbf{m}}\right) \cong \mathfrak{L} \bullet(\mathbf{m})$.

(2) The maximal reductive quotient of the stabilizer $\operatorname{Stab}_{G_{\beta}}\left(x_{\mathbf{m}}\right) \subset G_{\beta}$ of the point $x_{\mathbf{m}}$ is isomorphic to $G(\mathbf{m})$.

(3) The isomorphism in (1) induces the following commutative diagram:

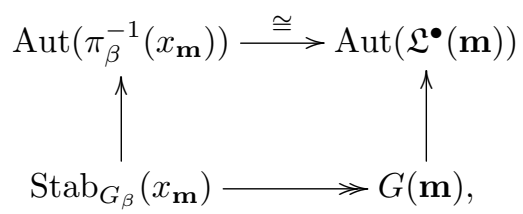

where the vertical arrows are the action maps and the lower horizontal arrow is the canonical quotient map in (2).

\section{Convolution and geometric extension algebras}

Let $\mathbb{k}$ be a field of characteristic zero. Later in Subsection 3.4, we specialize $\mathbb{k}=\mathbb{Q}(q)$. 


\subsection{Preliminary on equivariant geometry}

For the materials in this subsection, we refer [3] and [4].

Let $G$ be a complex linear algebraic group. A $G$-variety $X$ is a quasiprojective complex algebraic variety equipped with an algebraic action of the group $G$. We set pt $:=\operatorname{Spec} \mathbb{C}$ with the trivial $G$-action. The equivariant $K$ group $K^{G}(X)$ is defined to be the Grothendieck group of the abelian category of $G$-equivariant coherent sheaves on $X$ which is a module over the representation $\operatorname{ring} R(G)=K^{G}(\mathrm{pt})$. We put

$$
K^{G}(X)_{\mathbb{k}}:=K^{G}(X) \otimes_{\mathbb{Z}} \mathbb{k}, \quad R(G)_{\mathbb{k}}:=R(G) \otimes_{\mathbb{Z}} \mathbb{k} .
$$

Let $I \subset R(G)_{\mathbb{k}}$ be the augmentation ideal, i.e. the ideal generated by virtual representations of dimension 0 . We define the $I$-adic completions by

$$
\widehat{K}^{G}(X)_{\mathbb{k}}:=\underbrace{}_{k} \lim _{k} K^{G}(X)_{\mathbb{k}} / I^{k} K^{G}(X)_{\mathbb{k}}, \quad \widehat{R}(G)_{\mathbb{k}}:=\underbrace{\lim _{k}}_{k} R(G)_{\mathbb{k}} / I^{k} .
$$

The completed $K$-group $\widehat{K}^{G}(X)_{\mathbb{k}}$ is a module over the algebra $\widehat{R}(G)_{\mathbb{k}}$.

On the other hand, the $G$-equivariant Borel-Moore homology with $\mathbb{k}$-coefficients

$$
H_{*}^{G}(X, \mathbb{k})=\bigoplus_{k \in \mathbb{Z}} H_{k}^{G}(X, \mathbb{k}),
$$

is a module over the $G$-equivariant cohomology ring $H_{G}^{*}(\mathrm{pt}, \mathbb{k})$ of pt (with the cup product). Let us define the completion of a $\mathbb{Z}$-graded $\mathbb{k}$-vector space $V=$ $\bigoplus_{k \in \mathbb{Z}} V_{k}$ by $V^{\wedge}:=\prod_{k \in \mathbb{Z}} V_{k}$. The completion $H_{G}^{*}(\mathrm{pt}, \mathbb{k})^{\wedge}$ naturally becomes a $\mathbb{k}$-algebra and the completion $H_{*}^{G}(X, \mathbb{k})^{\wedge}$ becomes a module over $H_{G}^{*}(\mathrm{pt}, \mathbb{k})^{\wedge}$.

Assume that our $G$-variety $X$ is a $G$-stable closed subvariety of a non-singular ambient $G$-variety $M$. Then we have the $G$-equivariant local Chern character map

$$
\left(\operatorname{ch}^{G}\right)_{X}^{M}: \widehat{K}^{G}(X)_{\mathbb{k}} \rightarrow H_{*}^{G}(X, \mathbb{k})^{\wedge} .
$$

relative to $M$. We simply write $\mathrm{ch}^{G}$ instead of $\left(\mathrm{ch}^{G}\right)_{X}^{M}$ if the pair $(M, X)$ is obvious from the context. When $X=M=\mathrm{pt}$, the corresponding Chern character map induces an isomorphism of $\mathbb{k}$-algebras

$$
\widehat{R}(G)_{\mathbb{k}}=\widehat{K}^{G}(\mathrm{pt})_{\mathbb{k}} \cong H_{*}^{G}(\mathrm{pt}, \mathbb{k})^{\wedge}=H_{G}^{*}(\mathrm{pt}, \mathbb{k})^{\wedge} .
$$

We identify $H_{*}^{G}(\mathrm{pt}, \mathbb{k})^{\wedge}$ with $\widehat{R}(G)_{\mathbb{k}}$ via this isomorphism. Then $\left(\mathrm{ch}^{G}\right)_{X}^{M}$ is regarded as an $\widehat{R}(G)_{\mathbb{k}}$-homomorphism.

For a $G$-equivariant vector bundle $E$ on a non-singular $M$, let $\operatorname{Td}^{G}(E) \in$ $H_{G}^{*}(M, \mathbb{k})^{\wedge}$ be the $G$-equivariant Todd class. This is an invertible element with respect to the cup product. For the tangent bundle $T_{M}$ of $M$, we put $\operatorname{Td}_{M}^{G}:=$ $\operatorname{Td}^{G}\left(T_{M}\right)$.

Theorem 3.1 (Equivariant Riemann-Roch [4]). For $i=1,2$, let $X_{i}$ be a $G$ variety which is a $G$-stable closed subvariety of a non-singular ambient $G$-variety 
$M_{i}$. Assume that a $G$-equivariant morphism $\tilde{f}: M_{1} \rightarrow M_{2}$ restricts to a proper morphism $f: X_{1} \rightarrow X_{2}$. Then we have

$$
f_{*}\left(\operatorname{Td}_{M_{1}}^{G} \cdot\left(\operatorname{ch}^{G}\right)_{X_{1}}^{M_{1}}(\zeta)\right)=\operatorname{Td}_{M_{2}}^{G} \cdot\left(\operatorname{ch}^{G}\right)_{X_{2}}^{M_{2}}\left(f_{*} \zeta\right), \quad \zeta \in \widehat{K}^{G}\left(X_{1}\right)_{\mathbb{k}} .
$$

The following proposition is standard.

Proposition 3.2. Let $M$ be a non-singular $G$-variety. Let $Y \subset X \subset M$ be $G$-stable closed subvarieties, and $i: Y \hookrightarrow X, j: X \backslash Y \hookrightarrow X$ be inclusions. Then we have the following commutative diagram:

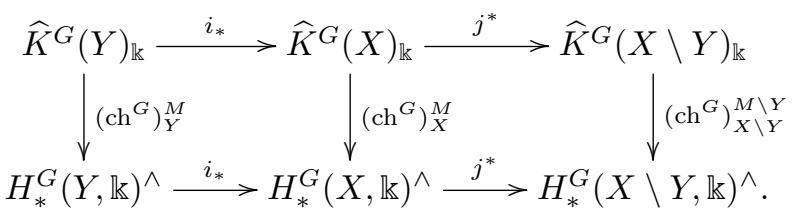

Next we consider the convolution products. Let $M_{i}$ be non-singular $G$ varieties for $i=1,2,3$. We denote by $p_{i j}: M_{1} \times M_{2} \times M_{3} \rightarrow M_{i} \times M_{j}$ the projection to the $(i, j)$-factors for $(i, j)=(1,2),(2,3),(1,3)$. Let $Z_{12} \subset M_{1} \times M_{2}$ and $Z_{23} \subset M_{2} \times M_{3}$ be $G$-stable closed subvarieties such that the morphism

$$
p_{13}: p_{12}^{-1}\left(Z_{12}\right) \cap p_{23}^{-1}\left(Z_{23}\right) \rightarrow Z_{13}:=p_{13}\left(p_{12}^{-1}\left(Z_{12}\right) \cap p_{23}^{-1}\left(Z_{23}\right)\right)
$$

is proper. Then we define the convolution product $*: K^{G}\left(Z_{12}\right) \otimes_{R(G)} K^{G}\left(Z_{23}\right) \rightarrow$ $K^{G}\left(Z_{13}\right)$ relative to $M_{1} \times M_{2} \times M_{3}$ by

$$
\zeta * \eta:=p_{13 *}\left(p_{12}^{*} \zeta \otimes_{M_{1} \times M_{2} \times M_{3}}^{\mathbb{L}} p_{23}^{*} \eta\right), \quad \zeta \in K^{G}\left(Z_{12}\right), \eta \in K^{G}\left(Z_{23}\right) .
$$

This naturally induces the convolution product on the completed $G$-equivariant $K$-groups $\widehat{K}^{G}\left(Z_{12}\right)_{\mathbb{k}} \otimes_{\widehat{R}(G)_{\mathrm{k}}} \widehat{K}^{G}\left(Z_{23}\right)_{\mathbb{k}} \rightarrow \widehat{K}^{G}\left(Z_{13}\right)_{\mathbb{k}}$. Similarly, we have the convolution product on the $G$-equivariant Borel-Moore homologies $H_{*}^{G}\left(Z_{12}, \mathbb{k}\right) \otimes_{H_{G}^{*}}(\mathrm{pt}, \mathbb{k})$ $H_{*}^{G}\left(Z_{23}, \mathbb{k}\right) \rightarrow H_{*}^{G}\left(Z_{13}, \mathbb{k}\right)$ relative to $M_{1} \times M_{2} \times M_{3}$ and its completed version $H_{*}^{G}\left(Z_{12}, \mathbb{k}\right)^{\wedge} \otimes_{\widehat{R}(G)_{\mathbf{k}}} H_{*}^{G}\left(Z_{23}, \mathbb{k}\right)^{\wedge} \rightarrow H_{*}^{G}\left(Z_{13}, \mathbb{k}\right)^{\wedge}$.

Under the situation in the previous paragraph, for each $(i, j)=(1,2),(2,3),(1,3)$, we also define the $G$-equivariant Riemann-Roch homomorphism $\mathrm{RR}^{G}: \widehat{K}^{G}\left(Z_{i j}\right)_{\mathbb{k}} \rightarrow$ $H_{*}^{G}\left(Z_{i j}, \mathbb{k}\right)^{\wedge}$ relative to $M_{i} \times M_{j}$ by

$$
\operatorname{RR}^{G}(\zeta):=\left(p_{i}^{*} \operatorname{Td}_{M_{i}}^{G}\right) \cdot\left(\operatorname{ch}^{G}\right)_{Z_{i j}}^{M_{i} \times M_{j}}(\zeta), \quad \zeta \in \widehat{K}^{G}\left(Z_{i j}\right)_{\mathbb{k}},
$$

where $p_{i}: M_{i} \times M_{j} \rightarrow M_{i}$ is the projection. By a completely similar discussion as in $[3,5.11 .11]$, we can prove the following.

Proposition 3.3. The $G$-equivariant Riemann-Roch homomorphisms are compatible with the convolution product, i.e. we have

$$
\operatorname{RR}^{G}(\zeta * \eta)=\operatorname{RR}^{G}(\zeta) * \operatorname{RR}^{G}(\eta), \quad \zeta \in \widehat{K}^{G}\left(Z_{12}\right)_{\mathbb{k}}, \eta \in \widehat{K}^{G}\left(Z_{23}\right)_{\mathbb{k}} .
$$




\subsection{Quiver Hecke algebra}

Fix an element $\beta=\sum_{i \in I} d_{i} \alpha_{i} \in \mathrm{Q}^{+}$and put $d:=\sum_{i \in I} d_{i}$. Let

$$
I^{\beta}:=\left\{\mathbf{i}=\left(i_{1}, \ldots, i_{d}\right) \in I^{d} \mid \alpha_{i_{1}}+\cdots+\alpha_{i_{d}}=\beta\right\} .
$$

The symmetric group $\mathfrak{S}_{d}$ of degree $d$ acts on the set $I^{\beta}$ from the right by $\left(i_{1}, \ldots, i_{d}\right) \cdot w:=\left(i_{w(1)}, \ldots, i_{w(d)}\right)$. Let $s_{k} \in \mathfrak{S}_{d}$ denote the transposition of $k$ and $k+1$ for $1 \leq k<d$.

Definition 3.4 (Khovanov-Lauda [16], Rouquier [20]). The quiver Hecke algebra $H_{Q}(\beta)$ is defined to be a $\mathbb{k}$-algebra with the generating set $\{e(\mathbf{i}) \mid \mathbf{i} \in$ $\left.I^{\beta}\right\} \cup\left\{x_{1}, \ldots, x_{d}\right\} \cup\left\{\tau_{1}, \ldots, \tau_{d-1}\right\}$, satisfying the following relations:

$$
\begin{gathered}
e(\mathbf{i}) e\left(\mathbf{i}^{\prime}\right)=\delta_{\mathbf{i}, \mathbf{i}^{\prime}} e(\mathbf{i}), \quad \sum_{\mathbf{i} \in I^{\beta}} e(\mathbf{i})=1, \quad x_{k} x_{l}=x_{l} x_{k}, \quad x_{k} e(\mathbf{i})=e(\mathbf{i}) x_{k}, \\
\tau_{k} e(\mathbf{i})=e\left(\mathbf{i} \cdot s_{k}\right) \tau_{k}, \quad \tau_{k} \tau_{l}=\tau_{l} \tau_{k} \quad \text { if }|k-l|>1, \\
\tau_{k}^{2} e(\mathbf{i})= \begin{cases}\left(x_{k}-x_{k+1}\right) e(\mathbf{i}), & \text { if } i_{k} \leftarrow i_{k+1}, \\
\left(x_{k+1}-x_{k}\right) e(\mathbf{i}), & \text { if } i_{k} \rightarrow i_{k+1}, \\
e(\mathbf{i}) & \text { if } a_{i_{k}, i_{k+1}}=0, \\
0 & \text { if } i_{k}=i_{k+1},\end{cases} \\
\left(\tau_{k} x_{l}-x_{s_{k}(l)} \tau_{k}\right) e(\mathbf{i})= \begin{cases}-e(\mathbf{i}) & \text { if } l=k, i_{k}=i_{k+1}, \\
e(\mathbf{i}) & \text { if } l=k+1, i_{k}=i_{k+1}, \\
0 & \text { otherwise, }\end{cases} \\
\left(\tau_{k+1} \tau_{k} \tau_{k+1}-\tau_{k} \tau_{k+1} \tau_{k}\right) e(\mathbf{i})= \begin{cases}e(\mathbf{i}) & \text { if } i_{k}=i_{k+2}, i_{k} \leftarrow i_{k+1}, \\
-e(\mathbf{i}) & \text { if } i_{k}=i_{k+2}, i_{k} \rightarrow i_{k+1}, \\
0 & \text { otherwise. }\end{cases}
\end{gathered}
$$

The quiver Hecke algebra $H_{Q}(\beta)$ is equipped with a $\mathbb{Z}$-grading given by

$$
\operatorname{deg} e(\mathbf{i})=0, \quad \operatorname{deg} x_{k}=2, \quad \operatorname{deg} \tau_{k} e(\mathbf{i})=-a_{i_{k}, i_{k+1}} .
$$

Since the grading is bounded from below (see [16, Theorem 2.5]), the completion $\widehat{H}_{Q}(\beta):=H_{Q}(\beta)^{\wedge}$ inherits a natural structure of $\mathbb{k}$-algebra.

We recall the faithful polynomial right representation of $H_{Q}(\beta)$ from [16, Section 2.3]. We set

$$
P_{\beta}:=\bigoplus_{\mathbf{i} \in I^{\beta}} \mathbb{k}\left[x_{1}, \ldots, x_{d}\right] 1_{\mathbf{i}}
$$

with a commutative $\mathbb{k}\left[x_{1}, \ldots, x_{d}\right]$-algebra structure $1_{\mathbf{i}} \cdot 1_{\mathbf{i}^{\prime}}=\delta_{\mathbf{i i}^{\prime}} 1_{\mathbf{i}}$. We define $f^{w}\left(x_{1}, \ldots, x_{d}\right):=f\left(x_{w(1)}, \ldots, x_{w(d)}\right)$ for $f \in \mathbb{k}\left[x_{1}, \ldots, x_{d}\right]$ and $w \in \mathfrak{S}_{d}$. 
Theorem 3.5 ([16] Proposition 2.3). The following formulas give a faithful right $H_{Q}(\beta)$-module structure on the $\mathbb{k}$-vector space $P_{\beta}$ :

$$
\begin{aligned}
a \cdot e(\mathbf{i}) & =a 1_{\mathbf{i}}, \\
a \cdot x_{k} & =a x_{k}, \\
\left(f 1_{\mathbf{i}}\right) \cdot \tau_{k} & = \begin{cases}\frac{f^{s_{k}}-f}{x_{k}-x_{k+1}} 1_{\mathbf{i}} & \text { if } i_{k}=i_{k+1}, \\
\left(x_{k+1}-x_{k}\right) f^{s_{k}} 1_{\mathbf{i} \cdot s_{k}} & \text { if } i_{k} \leftarrow i_{k+1}, \\
f^{s_{k}} 1_{\mathbf{i} \cdot s_{k}} & \text { otherwise, }\end{cases}
\end{aligned}
$$

where $a \in P_{\beta}$ and $f 1_{\mathbf{i}} \in \mathbb{k}\left[x_{1}, \ldots, x_{d}\right] 1_{\mathbf{i}}$.

Replacing the polynomial ring $\mathbb{k}\left[x_{1}, \ldots, x_{d}\right]$ with the ring $\mathbb{k} \llbracket x_{1}, \ldots, x_{d} \rrbracket$ of formal power series, we get the completion of the representation $P_{\beta}$ :

$$
\widehat{P}_{\beta}:=\bigoplus_{\mathbf{i} \in I^{\beta}} \mathbb{k} \llbracket x_{1}, \ldots, x_{d} \rrbracket 1_{\mathbf{i}}=P_{\beta} \otimes_{H_{Q}(\beta)} \widehat{H}_{Q}(\beta) .
$$

\subsection{Varagnolo-Vasserot's realization}

Fix an $I$-graded $\mathbb{C}$-vector space $D=\bigoplus_{i \in I} D_{i}$ with $\underline{\operatorname{dim}} D=\beta$, i.e. $\operatorname{dim} D_{i}=d_{i}$ as in Subsection 2.2. We consider the following two non-singular $G_{\beta}$-varieties:

$\mathcal{B}_{\beta}=\left\{F^{\bullet}=\left(D=F^{0} \supsetneq F^{1} \supsetneq \cdots \supsetneq F^{d}=0\right) \mid F^{k}\right.$ is an $I$-graded subspace of $\left.D\right\}$, $\mathcal{F}_{\beta}=\left\{\left(F^{\bullet}, x\right) \in \mathcal{B}_{\beta} \times E_{\beta} \mid x\left(F^{k}\right) \subset F^{k}\right.$ for any $\left.1 \leq k \leq d\right\}$.

The $G_{\beta}$-action on $\mathcal{F}_{\beta}$ is defined so that the projections $\mathrm{pr}_{1}: \mathcal{F}_{\beta} \rightarrow \mathcal{B}_{\beta}$ and $\mu_{\beta}:=\operatorname{pr}_{2}: \mathcal{F}_{\beta} \rightarrow E_{\beta}$ are $G_{\beta}$-equivariant. They decompose into connected components as

$$
\mathcal{B}_{\beta}=\bigsqcup_{\mathbf{i} \in I^{\beta}} \mathcal{B}_{\mathbf{i}}, \quad \mathcal{F}_{\beta}=\bigsqcup_{\mathbf{i} \in I^{\beta}} \mathcal{F}_{\mathbf{i}}
$$

where we put

$$
\mathcal{B}_{\mathbf{i}}:=\left\{F^{\bullet} \in \mathcal{B}_{\beta} \mid \underline{\operatorname{dim}} F^{k-1}=\underline{\operatorname{dim}} F^{k}+\alpha_{i_{k}}, \forall k\right\}, \quad \mathcal{F}_{\mathbf{i}}:=\left(\operatorname{pr}_{1}\right)^{-1}\left(\mathcal{B}_{\mathbf{i}}\right)
$$

for $\mathbf{i}=\left(i_{1}, \ldots, i_{d}\right) \in I^{\beta}$.

We fix a basis $\left\{v_{k}\right\}_{1 \leq k \leq d}$ of the vector space $D$ so that the set $\left\{v_{i, j}\right\}_{1 \leq j \leq d_{i}}$ forms a basis of the vector space $D_{i}$ for each $i \in I$, where we put $v_{i, j}:=$ $v_{d_{1}+\cdots+d_{i-1}+j}$. Let $H_{i} \subset G L\left(D_{i}\right)$ be the maximal torus fixing the lines $\left\{\mathbb{C} v_{i, j}\right\}_{1 \leq j \leq d_{i}}$ for each $i \in I$. We set $H_{\beta}:=\prod_{i \in I} H_{i} \subset G_{\beta}$.

Let $F_{0}^{\bullet} \in \mathcal{B}_{\beta}$ be the flag defined by $F_{0}^{k}:=\bigoplus_{l>k} \mathbb{C} v_{l}$, which belongs to the component $\mathcal{B}_{\mathbf{i}_{0}}$ with $\mathbf{i}_{0}:=\left(1^{d_{1}}, 2^{d_{2}}, \ldots, n^{d_{n}}\right) \in I^{\beta}$. For each $\mathbf{i} \in I^{\beta}$, we fix an element $w_{\mathbf{i}} \in \mathfrak{S}_{d}$ such that $\mathbf{i}=\mathbf{i}_{0} \cdot w_{\mathbf{i}}$. The set $\left\{w_{\mathbf{i}}\right\}_{\mathbf{i} \in I^{\beta}}$ forms a complete system of coset representatives for the quotient $\mathfrak{S}_{\beta} \backslash \mathfrak{S}_{d}$, where $\mathfrak{S}_{\beta}:=\operatorname{Stab}_{\mathfrak{S}_{d}}\left(\mathbf{i}_{0}\right)=$ $\mathfrak{S}_{d_{1}} \times \cdots \times \mathfrak{S}_{d_{n}}$. For each $w \in \mathfrak{S}_{d}$, we define the flag $F_{w}^{\bullet}$ by $F_{w}^{k}:=\bigoplus_{l>k} \mathbb{C} v_{w(l)}$ which belongs to the component $\mathcal{B}_{\mathbf{i}_{0} \cdot w}$. Let $F_{\mathbf{i}}^{\bullet}:=F_{w_{\mathbf{i}}}^{\bullet} \in \mathcal{B}_{\mathbf{i}}$ for $\mathbf{i} \in I^{\beta}$. Then we 
have $\mathcal{B}_{\mathbf{i}} \cong G_{\beta} / B_{\mathbf{i}}$ with $B_{\mathbf{i}}:=\operatorname{Stab}_{G_{\beta}}\left(F_{\mathbf{i}}\right) \subset G_{\beta}$ being the Borel subgroup fixing the flag $F_{\mathbf{i}}^{\bullet}$, which contains the maximal torus $H_{\beta}$. Then we have

$$
H_{*}^{G_{\beta}}\left(\mathcal{B}_{\mathbf{i}}, \mathbb{k}\right) \cong H_{*}^{B_{\mathrm{i}}}(\mathrm{pt}, \mathbb{k}) \cong H_{H_{\beta}}^{*}(\mathrm{pt}, \mathbb{k}) \cong \mathbb{k}\left[x_{1}, \ldots, x_{d}\right] 1_{\mathbf{i}},
$$

where the last isomorphism sends the 1 st $H_{\beta}$-equivariant Chern class of the line $\mathbb{C} v_{w_{\mathbf{i}}(k)}$ to the element $x_{k} 1_{\mathbf{i}}$. Thus we get an isomorphism

$$
H_{*}^{G_{\beta}}\left(\mathcal{B}_{\beta}, \mathbb{k}\right)=\bigoplus_{\mathbf{i} \in I^{\beta}} H_{*}^{G_{\beta}}\left(\mathcal{B}_{\mathbf{i}}, \mathbb{k}\right) \cong \bigoplus_{\mathbf{i} \in I^{\beta}} \mathbb{k}\left[x_{1}, \ldots, x_{d}\right] 1_{\mathbf{i}}=P_{\beta} .
$$

We consider the Steinberg type variety $\mathcal{Z}_{\beta}:=\mathcal{F}_{\beta} \times_{E_{\beta}} \mathcal{F}_{\beta}$ associated with the morphism $\mu_{\beta}: \mathcal{F}_{\beta} \rightarrow E_{\beta}$. Its $G_{\beta}$-equivariant Borel-Moore homology group $H_{*}^{G_{\beta}}\left(\mathcal{Z}_{\beta}, \mathbb{k}\right)$ becomes a $\mathbb{k}$-algebra with respect to the convolution product relative to $\mathcal{F}_{\beta} \times \mathcal{F}_{\beta} \times \mathcal{F}_{\beta}$. We identify the variety $\mathcal{B}_{\beta}$ with the fiber product $\{0\} \times_{E_{\beta}} \mathcal{F}_{\beta}$. Then the convolution product relative to $\{0\} \times \mathcal{F}_{\beta} \times \mathcal{F}_{\beta}$ makes the space $H_{*}^{G_{\beta}}\left(\mathcal{B}_{\beta}, \mathbb{k}\right)$ into a right $H_{*}^{G_{\beta}}\left(\mathcal{Z}_{\beta}, \mathbb{k}\right)$-module.

Let $\mu_{\mathbf{i}}$ denote the restriction of the proper morphism $\mu_{\beta}: \mathcal{F}_{\beta} \rightarrow E_{\beta}$ to the component $\mathcal{F}_{\mathbf{i}}$ for $\mathbf{i} \in I^{\beta}$. We put

$$
\mathcal{L}_{\beta}:=\bigoplus_{\mathbf{i} \in I^{\beta}}\left(\mu_{\mathbf{i}}\right)_{*} \mathbb{k}\left[\operatorname{dim} \mathcal{F}_{\mathbf{i}}\right]
$$

where $\mathbb{k}\left[\operatorname{dim} \mathcal{F}_{\mathbf{i}}\right]$ is the trivial local system (i.e. the constant $\mathbb{k}$-sheaf of rank 1 ) on $\mathcal{F}_{\mathbf{i}}$ homologically shifted by $\operatorname{dim} \mathcal{F}_{\mathbf{i}}$. By the decomposition theorem, we have

$$
\mathcal{L}_{\beta} \cong \bigoplus_{\mathbf{m} \in \mathrm{KP}(\beta)} L_{\mathbf{m}} \otimes_{\mathbb{k}} \mathcal{I} \mathcal{C}_{\mathbf{m}}=\bigoplus_{\mathbf{m} \in \operatorname{KP}(\beta)} \bigoplus_{k \in \mathbb{Z}} L_{\mathbf{m}, k} \otimes_{\mathbb{k}} \mathcal{I} \mathcal{C}_{\mathbf{m}}[k]
$$

where $\mathcal{I C}_{\mathbf{m}}$ denotes the intersection cohomology complex associated with the trivial local system on the orbit $\mathbb{O}_{\mathbf{m}}$ and $L_{\mathbf{m}}=\bigoplus_{k \in \mathbb{Z}} L_{\mathbf{m}, k}[k]$ is a self-dual finite-dimensional graded $\mathbb{k}$-vector space for each $\mathbf{m} \in \mathrm{KP}(\beta)$. The vector space $L_{\mathbf{m}}$ is known to be non-zero for all $\mathbf{m} \in \operatorname{KP}(\beta)$ (see [15, Corollary 2.8]). We consider the Yoneda algebra

$$
\operatorname{Ext}_{G_{\beta}}^{*}\left(\mathcal{L}_{\beta}, \mathcal{L}_{\beta}\right)=\bigoplus_{k \in \mathbb{Z}} \operatorname{Ext}_{G_{\beta}}^{k}\left(\mathcal{L}_{\beta}, \mathcal{L}_{\beta}\right)
$$

in the derived category of $G_{\beta}$-equivariant constructible complexes on $E_{\beta}$. This is a $\mathbb{Z}$-graded $\mathbb{k}$-algebra whose grading is bounded from below.

By a standard argument (see [3, Section 8.6]), we have an isomorphism of $\mathbb{k}$-algebras

$$
\operatorname{Ext}_{G_{\beta}}^{*}\left(\mathcal{L}_{\beta}, \mathcal{L}_{\beta}\right) \cong H_{*}^{G_{\beta}}\left(\mathcal{Z}_{\beta}, \mathbb{k}\right)
$$

Note that this is not compatible with the $\mathbb{Z}$-grading.

Let $\mathcal{L}_{\mathbf{i}}(k)$ be the $G_{\beta}$-equivariant line bundle on $\mathcal{F}_{\mathbf{i}}$ whose fiber at the point $\left(F^{\bullet}, x\right) \in \mathcal{F}_{\mathbf{i}}$ is $F^{k-1} / F^{k}$ for $\mathbf{i} \in I^{\beta}$ and $1 \leq k \leq d$. 
Theorem 3.6 (Varagnolo-Vasserot [21]). There is a unique isomorphism of $\mathbb{Z}$-graded $\mathbb{k}$-algebras

$$
H_{Q}(\beta) \stackrel{\cong}{\rightarrow} \operatorname{Ext}_{G_{\beta}}^{*}\left(\mathcal{L}_{\beta}, \mathcal{L}_{\beta}\right)
$$

which satisfies the following properties:

(1) The composition $H_{Q}(\beta) \stackrel{\cong}{\rightarrow} H^{G_{\beta}}\left(\mathcal{Z}_{\beta}, \mathbb{k}\right)$ of the isomorphisms (3.5) and (3.4) sends the element $e(\mathbf{i})$ (resp. $\left.x_{k} e(\mathbf{i})\right)$ to the push-forward of the fundamental class $\left[\mathcal{F}_{\mathbf{i}}\right]$ (resp. the 1 st $G_{\beta}$-equivariant Chern class of the line bundle $\mathcal{L}_{\mathbf{i}}(k)$ ) with respect to the diagonal embedding $\mathcal{F}_{\mathbf{i}} \rightarrow \mathcal{F}_{\mathbf{i}} \times_{E_{\beta}} \mathcal{F}_{\mathbf{i}}$;

(2) We have the following commutative diagram:

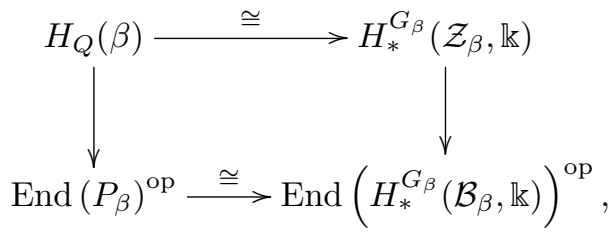

where the lower horizontal arrow denotes the isomorphism induced from (3.3) and the vertical arrows denote the right module structures.

Remark 3.7. Because our convention of the flag variety $\mathcal{B}_{\beta}$ differs from VaragnoloVasserot's [21], we need a modification. Actually, our isomorphism (3.5) is obtained by twisting the original isomorphism $H_{Q}(\beta) \cong \operatorname{Ext}_{G_{\beta}}^{*}\left(\mathcal{L}_{\beta}, \mathcal{L}_{\beta}\right)$ in [21] by a $\mathbb{k}$-algebra involution on $H_{Q}(\beta)$ given by

$$
e(\mathbf{i}) \mapsto e\left(\mathbf{i}^{\mathrm{op}}\right), \quad x_{k} \mapsto x_{d-k+1}, \quad \tau_{k} e(\mathbf{i}) \mapsto \begin{cases}-\tau_{d-k} e\left(\mathbf{i}^{\mathrm{op}}\right) & \text { if } i_{k}=i_{k+1} \\ \tau_{d-k} e\left(\mathbf{i}^{\mathrm{op}}\right) & \text { if } i_{k} \neq i_{k+1},\end{cases}
$$

where $\mathbf{i}^{\mathrm{op}}:=\left(i_{d}, \ldots, i_{2}, i_{1}\right)$ for $\mathbf{i}=\left(i_{1}, i_{2}, \ldots, i_{d}\right) \in I^{\beta}$.

Similarly to the case of the $G_{\beta}$-equivariant Borel-Moore homologies, the $K$-group $K^{G_{\beta}}\left(\mathcal{Z}_{\beta}\right)_{\mathbb{k}}$ becomes an $R\left(G_{\beta}\right)_{\mathbb{k}_{\mathbf{k}}}$-algebra and the $K$-group $K^{G_{\beta}}\left(\mathcal{B}_{\beta}\right)_{\mathbb{k}}$ becomes a right $K^{G_{\beta}}\left(\mathcal{Z}_{\beta}\right)_{\mathbb{k}}$-module with respect to the convolution products.

For each $\mathbf{i} \in I^{\beta}$, we have

$$
K^{G_{\beta}}\left(\mathcal{B}_{\mathbf{i}}\right)_{\mathbb{k}} \cong K^{B_{\mathrm{i}}}(\mathrm{pt})_{\mathbb{k}} \cong K^{H_{\beta}}(\mathrm{pt})_{\mathbb{k}}=R\left(H_{\beta}\right)_{\mathbb{k}} \cong \mathbb{k}\left[y_{1}^{ \pm 1}, \ldots, y_{d}^{ \pm 1}\right] 1_{\mathbf{i}}
$$

where the last isomorphism sends the class $\left[\mathbb{C} v_{w_{\mathbf{i}}(k)}\right]$ of the 1-dimensional $H_{\beta^{-}}$ module $\mathbb{C} v_{w_{\mathbf{i}}(k)}$ to the element $y_{k} 1_{\mathbf{i}}$. The $G_{\beta}$-equivariant Chern character map $\left(\mathrm{ch}^{G_{\beta}}\right)_{\mathcal{B}_{\mathrm{i}}}^{\mathcal{B}_{\mathrm{i}}}$ gives an isomorphism of $\mathbb{k}$-algebras

$$
\widehat{K}^{G_{\beta}}\left(\mathcal{B}_{\mathbf{i}}\right)_{\mathbb{k}} \cong \mathbb{k} \llbracket y_{1}-1, \ldots, y_{d}-1 \rrbracket 1_{\mathbf{i}} \cong \mathbb{\cong} \llbracket x_{1}, \ldots, x_{d} \rrbracket 1_{\mathbf{i}} \cong H_{*}^{G_{\beta}}\left(\mathcal{B}_{\mathbf{i}}, \mathbb{k}\right)^{\wedge},
$$

where the middle arrow sends the element $y_{k} 1_{\mathbf{i}}$ to the exponential $e^{x_{k}} 1_{\mathbf{i}}$ for $1 \leq k \leq d$. Applying the equivariant Riemann-Roch theorem (=Theorem 3.1) to the inclusion $\mathcal{B}_{\mathbf{i}} \hookrightarrow \mathcal{F}_{\mathbf{i}}$, we have

$$
\left(\operatorname{ch}^{G_{\beta}}\right)_{\mathcal{B}_{\mathbf{i}}}^{\mathcal{F}_{\mathbf{i}}}=C_{\mathbf{i}} \cdot\left(\operatorname{ch}^{G_{\beta}}\right)_{\mathcal{B}_{\mathbf{i}}}^{\mathcal{B}_{\mathbf{i}}}, \quad C_{\mathbf{i}}:=\left(\operatorname{Td}_{\mathcal{F}_{\mathbf{i}}}^{G_{\beta}}\right)^{-1} \operatorname{Td}_{\mathcal{B}_{\mathbf{i}}}^{G_{\beta}} \cdot 1_{\mathbf{i}} \in \mathbb{k} \llbracket x_{1}, \ldots, x_{d} \rrbracket 1_{\mathbf{i}}
$$


and hence the map $\left(\mathrm{ch}^{G_{\beta}}\right)_{\mathcal{B}_{\mathbf{i}}}^{\mathcal{F}_{\mathbf{i}}}$ is an isomorphism of $\widehat{R}\left(G_{\beta}\right)_{\mathbb{k}_{\mathbf{k}}}$-modules. Summing up over $\mathbf{i} \in I^{\beta}$, we obtain an isomorphism of $\widehat{R}\left(G_{\beta}\right)_{\mathbb{k}}$-modules

$$
\left(\mathrm{ch}^{G_{\beta}}\right)_{\mathcal{B}_{\beta}}^{\mathcal{F}_{\beta}}: \widehat{K}^{G_{\beta}}\left(\mathcal{B}_{\beta}\right)_{\mathbb{k}} \cong H_{*}^{G_{\beta}}\left(\mathcal{B}_{\beta}, \mathbb{k}\right)^{\wedge}
$$

Proposition 3.8. The Riemann-Roch homomorphism gives an isomorphism of $\widehat{R}\left(G_{\beta}\right)_{\mathbb{k}}$-algebras:

$$
\operatorname{RR}^{G_{\beta}}: \widehat{K}^{G_{\beta}}\left(\mathcal{Z}_{\beta}\right)_{\mathbb{k}} \cong H_{*}^{G_{\beta}}\left(\mathcal{Z}_{\beta}, \mathbb{k}\right)^{\wedge}
$$

which makes the following diagram commute:

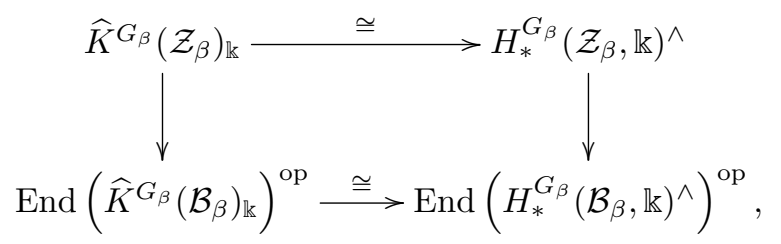

where the lower horizontal arrow denotes the isomorphism induced from (3.7) and the vertical arrows denote the right module structures.

Proof. By Proposition 3.3, the map $\mathrm{RR}^{G_{\beta}}: \widehat{K}^{G_{\beta}}\left(\mathcal{Z}_{\beta}\right)_{\mathrm{k}} \rightarrow H_{*}^{G_{\beta}}\left(\mathcal{Z}_{\beta}, \mathbb{k}\right)^{\wedge}$ is an algebra homomorphism and the diagram (3.8) commutes. To prove that the map $\mathrm{RR}^{G_{\beta}}: \widehat{K}^{G_{\beta}}\left(\mathcal{Z}_{\beta}\right)_{\mathbb{k}} \rightarrow H_{*}^{G_{\beta}}\left(\mathcal{Z}_{\beta}, \mathbb{k}\right)^{\wedge}$ is an isomorphism, it suffices to check that the equivariant Chern character map $\left(\operatorname{ch}^{G_{\beta}}\right)_{\mathcal{Z}_{\beta}}^{\mathcal{F}_{\beta} \times \mathcal{F}_{\beta}}: \widehat{K}^{G_{\beta}}\left(\mathcal{Z}_{\beta}\right)_{\mathbb{k}} \rightarrow$ $H_{*}^{G_{\beta}}\left(\mathcal{Z}_{\beta}, \mathbb{k}\right)^{\wedge}$ gives an isomorphism of $\widehat{R}\left(G_{\beta}\right)_{\mathbb{k}}$-modules since $\mathrm{RR}^{G_{\beta}}$ is obtained from $\left(\mathrm{ch}^{G_{\beta}}\right)_{\mathcal{Z}_{\beta}}^{\mathcal{F}_{\beta} \times \mathcal{F}_{\beta}}$ by multiplying the $G_{\beta}$-equivariant Todd class $p_{1}^{*} \operatorname{Td}_{\mathcal{F}_{\beta}}^{G_{\beta}}$, which is an invertible element. Because we have the connected component decomposition

$$
\mathcal{Z}_{\beta}=\bigsqcup_{\mathbf{i}, \mathbf{i}^{\prime} \in I^{\beta}} \mathcal{Z}_{\mathbf{i}, \mathbf{i}^{\prime}}, \quad \mathcal{Z}_{\mathbf{i}, \mathbf{i}^{\prime}}:=\mathcal{F}_{\mathbf{i}} \times_{E_{\beta}} \mathcal{F}_{\mathbf{i}^{\prime}}
$$

we focus on a connected component

$$
\mathcal{Z}_{\mathbf{i}, \mathbf{i}^{\prime}}=\left\{\left(F^{\bullet}, F^{\prime \bullet}, x\right) \in \mathcal{B}_{\mathbf{i}} \times \mathcal{B}_{\mathbf{i}^{\prime}} \times E_{\beta} \mid x\left(F^{k}\right) \subset F^{k}, x\left(F^{\prime k}\right) \subset F^{\prime k}, \forall k\right\} .
$$

For each $w \in \mathfrak{S}_{\beta} w_{\mathbf{i}^{\prime}}$, we define a locally closed $G_{\beta}$-subvariety

$$
\mathcal{Z}_{\mathbf{i}, \mathbf{i}^{\prime}}^{w}=G_{\beta} \times^{B_{\mathbf{i}}}\left\{\left(F_{\mathbf{i}}^{\bullet}, F^{\prime \bullet}, x\right) \in \mathcal{Z}_{\mathbf{i}, \mathbf{i}^{\prime}} \mid F^{\prime \bullet} \in B_{\mathbf{i}} F_{w}^{\bullet}\right\}
$$

which is a $G_{\beta}$-equivariant affine bundle over $\mathcal{B}_{\mathbf{i}}$. They give a $G_{\beta}$-stable stratification $\mathcal{Z}_{\mathbf{i}, \mathbf{i}^{\prime}}:=\bigsqcup_{w \in \mathfrak{S}_{\beta} w_{\mathbf{i}^{\prime}}} Z_{\mathbf{i}, \mathbf{i}^{\prime}}^{w}$. Fix a total ordering $\mathfrak{S}_{\beta} w_{\mathbf{i}^{\prime}}=\left\{w_{1}, w_{2}, \ldots, w_{m}\right\}$ such that we have $w_{k} w_{\mathbf{i}}^{-1}<w_{l} w_{\mathbf{i}}^{-1}$ in the Bruhat ordering only if $k<l$. We simply write $\mathcal{Z}_{\mathbf{i}, \mathbf{i}^{\prime}}^{k}:=\mathcal{Z}_{\mathbf{i}, \mathbf{i}^{\prime}}^{w_{k}}$ and set $\mathcal{Z}_{\mathbf{i}, \mathbf{i}^{\prime}}^{\leq k}:=\bigsqcup_{j \leq k} \mathcal{Z}_{\mathbf{i}, \mathbf{i}^{\prime}}^{j}$. Then for each $k$, the variety $\mathcal{Z}_{\mathbf{i}, \mathbf{i}^{\prime}}^{\leq k-1}$ is closed in $\mathcal{Z}_{\mathbf{i}, \mathbf{i}^{\prime}}^{\leq k}$ and its complement is $\mathcal{Z}_{\mathbf{i}, \mathbf{i}^{\prime}}^{k}$. Since $\mathcal{Z}_{\mathbf{i}, \mathbf{i}^{\prime}}^{k}$ is a $G_{\beta^{-}}$-equivariant affine bundle over $\mathcal{B}_{\mathbf{i}}$, its homology of odd degree vanishes: 
$H_{\text {odd }}^{G_{\beta}}\left(\mathcal{Z}_{\mathbf{i}, \mathrm{i}^{\prime}}^{k}, \mathbb{k}\right)=0$. Therefore an inductive argument with respect to $k$ yields $H_{\text {odd }}^{G_{\beta}}\left(\mathcal{Z}_{\mathbf{i}, \mathbf{i}^{\prime}}^{\leq k}, \mathbb{k}\right)=0$. Using the cellular fibration lemma [3, 5.5.1] for equivariant $K$-groups and Proposition 3.2, we obtain the following commutative diagram with exact rows for each $k$ :

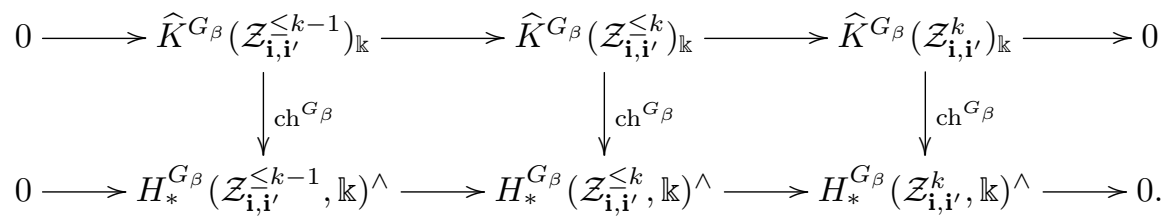

Note that the map $\operatorname{ch}^{G_{\beta}}: \widehat{K}^{G_{\beta}}\left(\mathcal{Z}_{\mathbf{i}, \mathbf{i}^{\prime}}^{k}\right)_{\mathbb{k}} \rightarrow H_{*}^{G_{\beta}}\left(\mathcal{Z}_{\mathbf{i}, \mathbf{i}^{\prime}}^{k}, \mathbb{k}\right)^{\wedge}$ is an isomorphism for any $k$ since again the variety $Z_{\mathbf{i}, \mathbf{i}^{\prime}}^{k}$ is an affine bundle over $\mathcal{B}_{\mathbf{i}}$. Hence, by induction on $k$, we conclude that $\operatorname{ch}^{G_{\beta}}: \widehat{K}^{G_{\beta}}\left(\mathcal{Z}_{\mathbf{i}, \mathbf{i}^{\prime}}^{\leq k}\right)_{\mathbb{k}} \rightarrow H_{*}^{G_{\beta}}\left(\mathcal{Z}_{\mathbf{i}, \mathbf{i}^{\prime}}^{\leq k}, \mathbb{k}\right)^{\wedge}$ is an isomorphism for all $k$.

Note that the isomorphism (3.4) induces an isomorphism between the completions:

$$
\operatorname{Ext}_{G_{\beta}}^{*}\left(\mathcal{L}_{\beta}, \mathcal{L}_{\beta}\right)^{\wedge} \cong H_{*}^{G_{\beta}}\left(\mathcal{Z}_{\beta}, \mathbb{k}\right)^{\wedge} .
$$

As a summary of this subsection, we have the following.

Corollary 3.9. We have the following isomorphisms of $\mathbb{k}$-algebras:

$$
\widehat{H}_{Q}(\beta) \cong \operatorname{Ext}_{G_{\beta}}^{*}\left(\mathcal{L}_{\beta}, \mathcal{L}_{\beta}\right)^{\wedge} \cong H_{*}^{G_{\beta}}\left(\mathcal{Z}_{\beta}, \mathbb{k}\right)^{\wedge} \cong \widehat{K}^{G_{\beta}}\left(\mathcal{Z}_{\beta}\right)_{\mathbb{k}} .
$$

\subsection{Nakajima's homomorphism and the category $\mathcal{C}_{Q, \beta}$}

Henceforth, we specialize $\mathbb{k}$ to be the field $\mathbb{Q}(q)$ of rational functions in an indeterminate $q$. In this subsection, we consider the quantum loop algebra $U_{q} \equiv U_{q}(L \mathfrak{g})$ defined over $\mathbb{k}$. The quantum loop algebra $U_{q}(L \mathfrak{g})$ is isomorphic to the level zero quotient of the quantum affine algebra $U_{q}^{\prime}(\widehat{\mathfrak{g}})$ without the degree operator. We do not recall the definitions here. See e.g. [5], [10], [17] for the precise definitions of $U_{q}(L \mathfrak{g})$ or $U_{q}^{\prime}(\widehat{\mathfrak{g}})$.

Recall the quiver varieties with proper $\mathbb{G}(\lambda)$-equivariant morphism $\pi: \mathfrak{M}(\lambda) \rightarrow$ $\mathfrak{M}_{0}(\lambda)$ for each $\lambda \in \mathrm{P}^{+}$(see Subsection 2.4). We consider the Steinberg type variety $Z(\lambda):=\mathfrak{M}(\lambda) \times_{\mathfrak{M}_{0}(\lambda)} \mathfrak{M}(\lambda)$. Then its $\mathbb{G}(\lambda)$-equivariant $K$-group $K^{\mathbb{G}(\lambda)}(Z(\lambda))$ becomes an $R(\mathbb{G}(\lambda)$ )-algebra with respect to the convolution product relative to $\mathfrak{M}(\lambda) \times \mathfrak{M}(\lambda) \times \mathfrak{M}(\lambda)$. We identify the fiber $\mathfrak{L}(\lambda)=\pi^{-1}(0)$ with the fiber product $\mathfrak{M}(\lambda) \times_{\mathfrak{M}_{0}(\lambda)}\{0\}$. Then the convolution product relative to $\mathfrak{M}(\lambda) \times \mathfrak{M}(\lambda) \times\{0\}$ makes the $K$-group $K^{\mathbb{G}(\lambda)}(\mathfrak{L}(\lambda))$ into a left $K^{\mathbb{G}(\lambda)}(Z(\lambda))$-module.

Recall that $\mathbb{G}(\lambda)=G(\lambda) \times \mathbb{C}^{\times}$. We set $A:=R\left(\mathbb{C}^{\times}\right)$and identify $A=\mathbb{Z}\left[v^{ \pm 1}\right]$ in the standard way. Specializing $v$ to $q$, we regard $\mathbb{k}$ as an $A$-algebra.

Theorem 3.10 (Nakajima [17] Theorem 9.4.1). There exists a $\mathbb{k}$-algebra homomorphism

$$
\Phi_{\lambda}: U_{q}(L \mathfrak{g}) \rightarrow K^{\mathbb{G}(\lambda)}(Z(\lambda)) \otimes_{A} \mathbb{k}
$$


such that the pull-back

$$
\mathbb{W}(\lambda):=\Phi_{\lambda}^{*}\left(K^{\mathbb{G}(\lambda)}(\mathfrak{L}(\lambda)) \otimes_{A} \mathbb{k}\right)
$$

is a cyclic $U_{q}(L \mathfrak{g})$-module generated by an extremal weight vector $w_{\lambda}:=\left[\mathcal{O}_{\mathfrak{L}(0, \lambda)}\right] \in$ $K^{\mathbb{G}(\lambda)}(\mathfrak{L}(0, \lambda)) \otimes_{A} \mathbb{k}$ of weight $\lambda$. Moreover the module $\mathbb{W}(\lambda)$ is free of finite rank over $\operatorname{End}_{U_{q}}(\mathbb{W}(\lambda)) \cong R(\mathbb{G}(\lambda)) \otimes_{A} \mathbb{k}$.

Remark 3.11. The module $\mathbb{W}(\lambda)$ is known to be isomorphic to the global Weyl module defined by Chari-Pressley [2] and also to the level 0 extremal weight module defined by Kashiwara [13]. In particular, if $\lambda=\varpi_{i}$ for some $i \in I$, the module $\mathbb{W}\left(\varpi_{i}\right)$ is isomorphic to the affinization of the fundamental module $W\left(\varpi_{i}\right)$ (see [14]).

Take an element $\boldsymbol{\lambda} \in \mathscr{P}^{+}$with $\operatorname{cl}(\boldsymbol{\lambda})=\lambda$ and recall the 1-dimensional subtorus $T(\boldsymbol{\lambda}) \subset \mathbb{G}(\boldsymbol{\lambda}) \subset \mathbb{G}(\lambda)$. We identify $R(T(\boldsymbol{\lambda}))=A$ via the isomorphism $\prod_{i \in I} f_{i} \times$ id : $\mathbb{C}^{\times} \stackrel{\cong}{\rightarrow} T(\boldsymbol{\lambda})$. Let $\mathfrak{m}_{\boldsymbol{\lambda}}$ be the kernel of the restriction $R(\mathbb{G}(\lambda)) \otimes_{A} \mathbb{k} \rightarrow R(T(\boldsymbol{\lambda})) \otimes_{A} \mathbb{k}=\mathbb{k}$. The corresponding specialization $\mathbb{W}(\lambda) / \mathfrak{m}_{\boldsymbol{\lambda}} \mathbb{W}(\lambda)$ (known as the local Weyl module defined in [2]) has a unique simple quotient $L(\boldsymbol{\lambda})$ in $U_{q}$ - $\bmod _{\mathrm{fd}}$.

Definition 3.12 (Hernandez-Leclerc [8]). We define the category $\mathcal{C}_{Q}$ (resp. $\mathcal{C}_{Q, \beta}$ for each $\beta \in \mathrm{Q}^{+}$) to be the minimal Serre full subcategory of the category $U_{q}$ - $\bmod _{\mathrm{fd}}$ of finite-dimensional $U_{q}(L \mathfrak{g})$-modules containing the simple objects $\left\{L(\boldsymbol{\lambda}) \mid \boldsymbol{\lambda} \in \mathscr{P}_{0}^{+}\right\}$(resp. $\left.\{L(\mathbf{m}) \mid \mathbf{m} \in \mathrm{KP}(\beta)\}\right)$, where $\mathscr{P}_{0}^{+}=\bigsqcup_{\beta \in \mathrm{Q}^{+}} \operatorname{KP}(\beta) \subset$ $\mathscr{P}^{+}$is as in Subsection 2.5.

Remark 3.13. Let $G$ be a linear algebraic group whose Lie algebra is $\mathfrak{g}$ and $N$ be the maximal unipotent subgroup of $G$ corresponding to the positive roots. Hernandez-Leclerc [8] proved that the category $\mathcal{C}_{Q}$ is a monoidal subcategory and there is an isomorphism from the complexified Grothendieck ring $K\left(\mathcal{C}_{Q}\right)_{\mathbb{C}}$ to the coordinate ring $\mathbb{C}[N]$, which sends the classes of simple objects to the elements of the dual canonical basis bijectively. Actually, Hernandez-Leclerc established an isomorphism between their quantizations. We have a block decomposition $\mathcal{C}_{Q}=\bigoplus_{\beta \in \mathrm{Q}^{+}} \mathcal{C}_{Q, \beta}$ satisfying $\mathcal{C}_{Q, \beta} \otimes \mathcal{C}_{Q, \beta^{\prime}} \subset \mathcal{C}_{Q, \beta+\beta^{\prime}}$ (see [5, Section 2.6]). This decomposition corresponds to the weight decomposition $\mathbb{C}[N]=\bigoplus_{\beta \in Q^{+}} \mathbb{C}[N]_{\beta}$. The isomorphism $\mathfrak{M}_{0, \beta}^{\bullet} \cong E_{\beta}$ in Theorem 2.1 was originally established in order to give a geometric interpretation to the isomorphism $K\left(\mathcal{C}_{Q, \beta}\right)_{\mathbb{C}} \cong \mathbb{C}[N]_{\beta}$.

Now we fix an element $\beta \in \mathrm{Q}^{+}$. In Subsection 2.5, we defined the graded quiver variety $\mathfrak{M}_{\beta}^{\bullet}$ with a canonical $\mathbb{G}_{\beta}$-equivariant proper morphism $\pi_{\beta}: \mathfrak{M}_{\beta}^{\bullet} \rightarrow$ $E_{\beta}$, which is obtained from $\pi: \mathfrak{M}(\lambda) \rightarrow \mathfrak{M}_{0}(\lambda)$ with $\lambda=\operatorname{cl}\left(\boldsymbol{\lambda}_{\beta}\right)$ by taking the fixed locus with respect to the action of the 1-dimensional torus $T_{\beta} \subset \mathbb{G}_{\beta} \subset$ $\mathbb{G}(\lambda)$. We form the Steinberg type variety $Z_{\beta}^{\bullet}:=\mathfrak{M}_{\beta}^{\bullet} \times_{E_{\beta}} \mathfrak{M}_{\beta}^{\bullet}=Z(\lambda)^{T_{\beta}}$. Let $\mathfrak{r}_{\beta}$ be the kernel of the restriction $R\left(\mathbb{G}_{\beta}\right) \otimes_{A} \mathbb{k} \rightarrow R\left(T_{\beta}\right) \otimes_{A} \mathbb{k}=\mathbb{k}$. Note that the decomposition (2.2) $\mathbb{G}_{\beta} \cong G_{\beta} \times T_{\beta}$ yields an isomorphism

$$
K^{\mathbb{G}_{\beta}}(X) \otimes_{A} \mathbb{k} \cong K^{G_{\beta}}(X)_{\mathbb{k}}
$$


for any $\mathbb{G}_{\beta}$-variety $X$ with a trivial $T_{\beta}$-action. In particular, we have an isomorphism $R\left(\mathbb{G}_{\beta}\right) \otimes_{A} \mathbb{k} \cong R\left(G_{\beta}\right)_{\mathbb{k}}$ of $\mathbb{k}$-algebras, via which the maximal ideal $\mathfrak{r}_{\beta} \subset R\left(\mathbb{G}_{\beta}\right) \otimes_{A} \mathbb{k}$ corresponds to the augmentation ideal $I \subset R\left(G_{\beta}\right)_{\mathbb{k}}$. Therefore we have an isomorphism

$$
\left[K^{\mathbb{G}_{\beta}}(X) \otimes_{A} \mathbb{k}\right]_{\mathfrak{r}_{\beta}}^{\wedge} \cong \widehat{K}^{G_{\beta}}(X)_{\mathbb{k}},
$$

where $[-]_{\mathfrak{r}_{\beta}}^{\wedge}$ denotes the $\mathfrak{r}_{\beta}$-adic completion. We define the $\mathbb{k}$-algebra homomorphism $\widehat{\Phi}_{\beta}: U_{q}(L \mathfrak{g}) \rightarrow \widehat{K}^{G_{\beta}}\left(Z_{\beta}^{\bullet}\right)_{\mathrm{k}}$ as the following composition:

$$
\begin{aligned}
U_{q}(L \mathfrak{g}) & \stackrel{\Phi_{\lambda}}{\longrightarrow} K^{\mathbb{G}(\lambda)}(Z(\lambda)) \otimes_{A} \mathbb{k} & & \\
& \rightarrow K^{\mathbb{G}_{\beta}}(Z(\lambda)) \otimes_{A} \mathbb{k} & & \left(\text { restriction to } \mathbb{G}_{\beta} \subset \mathbb{G}(\lambda)\right) \\
& \rightarrow\left[K^{\mathbb{G}_{\beta}}(Z(\lambda)) \otimes_{A} \mathbb{k}\right]_{\mathfrak{r}_{\beta}}^{\wedge} & & \left(\mathfrak{r}_{\beta}\right. \text {-adic completion) } \\
& \cong\left[K^{\mathbb{G}_{\beta}}\left(Z_{\beta}^{\bullet}\right) \otimes_{A} \mathbb{k}\right]_{\mathfrak{r}_{\beta}}^{\wedge} & & \text { (localization theorem) } \\
& \cong \widehat{K}^{G_{\beta}}\left(Z_{\beta}^{\bullet}\right)_{\mathbb{k}} . & & \text { (isomorphism (3.9)) }
\end{aligned}
$$

Theorem 3.14 ([5] Theorem 4.9). The pull-back along the homomorphism $\widehat{\Phi}_{\beta}: U_{q}(L \mathfrak{g}) \rightarrow \widehat{K}^{G_{\beta}}\left(Z_{\beta}^{\bullet}\right)_{\mathbb{k}}$ induces an equivalence

$$
\widehat{\Phi}_{\beta}^{*}: \widehat{K}^{G_{\beta}}\left(Z_{\beta}^{\bullet}\right)_{\mathbb{k}}-\bmod _{\mathrm{fd}} \stackrel{\simeq}{\rightarrow} \mathcal{C}_{Q, \beta}
$$

between the category $\widehat{K}^{G_{\beta}}\left(Z_{\beta}^{\bullet}\right)_{\mathbb{k}}-\bmod _{\mathrm{fd}}$ of finite-dimensional $\widehat{K}^{G_{\beta}}\left(Z_{\beta}^{\bullet}\right)_{\mathbb{k}}$-modules and the category $\mathcal{C}_{Q, \beta} \subset U_{q}$-mod $\bmod _{\mathrm{fd}}$.

The next proposition is a counterpart of Proposition 3.8.

Proposition 3.15. The Riemann-Roch homomorphism gives an isomorphism of $\widehat{R}\left(G_{\beta}\right)_{\mathrm{k}}$-algebras:

$$
\mathrm{RR}^{G_{\beta}}: \widehat{K}^{G_{\beta}}\left(Z_{\beta}^{\bullet}\right)_{\mathbb{k}} \cong H_{*}^{G_{\beta}}\left(Z_{\beta}^{\bullet}, \mathbb{k}\right)^{\wedge} .
$$

Proof. As in the proof of Proposition 3.8, it suffices to prove that the equivariant Chern character map $\left(\operatorname{ch}^{G_{\beta}}\right)_{Z_{\beta}^{\bullet}}^{\mathfrak{M}_{\beta}^{\bullet} \times \mathfrak{M}_{\beta}^{\bullet}}: \widehat{K}^{G_{\beta}}\left(Z_{\beta}^{\bullet}\right)_{\mathbb{k}} \rightarrow H_{*}^{G_{\beta}}\left(Z_{\beta}^{\bullet}, \mathbb{k}\right)^{\wedge}$ is an isomorphism.

Note that the $G_{\beta}$-orbit stratification (2.1) yields a stratification of $Z_{\beta}^{\bullet}$ :

$$
Z_{\beta}^{\bullet}=\left.\bigsqcup_{\mathbf{m} \in \operatorname{KP}(\beta)} Z_{\beta}^{\bullet}\right|_{\mathbb{O}_{\mathbf{m}}},\left.\quad Z_{\beta}^{\bullet}\right|_{\mathbb{O}_{\mathbf{m}}} \cong G_{\beta} \times{ }^{\operatorname{Stab}_{G_{\beta}}\left(x_{\mathbf{m}}\right)}\left(\pi_{\beta}^{-1}\left(x_{\mathbf{m}}\right) \times \pi_{\beta}^{-1}\left(x_{\mathbf{m}}\right)\right) .
$$

Fix a total ordering $\operatorname{KP}(\beta)=\left\{\mathbf{m}_{1}, \mathbf{m}_{2}, \ldots, \mathbf{m}_{s}\right\}$ such that we have $\mathbb{O}_{k} \subset \overline{\mathbb{O}}_{l}$ only if $k<l$. Set $Z_{\beta}^{k}:=\left.Z_{\beta}^{\bullet}\right|_{\mathbb{Q}_{\mathbf{m}_{k}}}$ and $Z_{\beta}^{\leq k}:=\bigsqcup_{j \leq k} Z_{\beta}^{j}$. Then the variety $Z_{\beta}^{\leq k-1}$ is a closed subvariety of $Z_{\beta}^{\leq k}$ whose complement is $Z_{\beta}^{k}$. By Proposition 2.2 and the reduction, we have

$$
\begin{aligned}
K^{G_{\beta}}\left(Z_{\beta}^{k}\right) & \cong K^{G\left(\mathbf{m}_{k}\right)}\left(\mathfrak{L}^{\bullet}\left(\mathbf{m}_{k}\right) \times \mathfrak{L} \bullet\left(\mathbf{m}_{k}\right)\right), \\
H_{*}^{G_{\beta}}\left(Z_{\beta}^{k}, \mathbb{k}\right) & \cong H_{*}^{G\left(\mathbf{m}_{k}\right)}\left(\mathfrak{L}^{\bullet}\left(\mathbf{m}_{k}\right) \times \mathfrak{L} \bullet\left(\mathbf{m}_{k}\right), \mathbb{k}\right)
\end{aligned}
$$


for each $k$. Then, using [17, Theorem 7.4.1], we can prove that the equivariant Chern character map gives an isomorphism $\mathrm{ch}^{G_{\beta}}: \widehat{K}^{G_{\beta}}\left(Z_{\beta}^{k}\right)_{\mathbf{k}} \cong H_{*}^{G_{\beta}}\left(Z_{\beta}^{k}, \mathbb{k}\right)^{\wedge}$ for each $k$. Moreover, we obtain the following commutative diagram with exact rows for each $k$ :

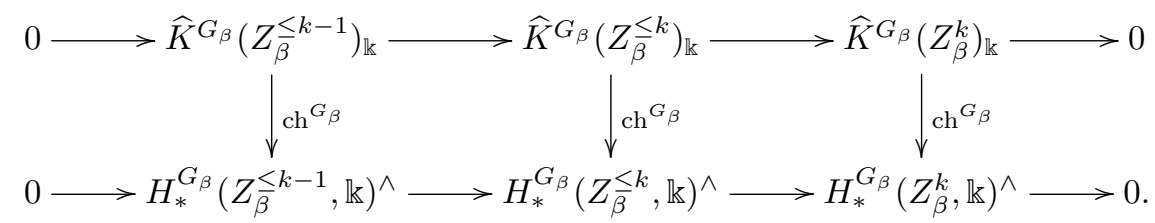

By induction on $k$, the equivariant Chern character map gives an isomorphism $\operatorname{ch}^{G_{\beta}}: \widehat{K}^{G_{\beta}}\left(Z_{\bar{\beta}}^{\leq k}\right)_{\mathbb{k}} \cong H_{*}^{G_{\beta}}\left(Z_{\bar{\beta}}^{\leq k}, \mathbb{k}\right)^{\wedge}$ for all $k$.

We consider the proper push-forward

$$
\mathcal{L}_{\beta}^{\bullet}:=\left(\pi_{\beta}\right)_{*} \underline{\mathbb{k}}
$$

of the trivial local system $\underline{\mathbb{k}}$ on $\mathfrak{M}_{\beta}^{\bullet}$. By the decomposition theorem, we have

$$
\mathcal{L}_{\beta}^{\bullet} \cong \bigoplus_{\mathbf{m} \in \operatorname{KP}(\beta)} L_{\mathbf{m}}^{\bullet} \otimes_{\mathbb{k}} \mathcal{I} \mathcal{C}_{\mathbf{m}}=\bigoplus_{\mathbf{m} \in \operatorname{KP}(\beta)} \bigoplus_{k \in \mathbb{Z}} L_{\mathbf{m}, k}^{\bullet} \otimes_{\mathbb{k}} \mathcal{I} \mathcal{C}_{\mathbf{m}}[k]
$$

where $L_{\mathbf{m}}^{\bullet}=\bigoplus_{k} L_{\mathbf{m}, k}^{\bullet}$ is a finite-dimensional graded $\mathbb{k}$-vector space, which is known to be non-zero for each $\mathbf{m}$ (see [17, Theorem 14.3.2]). Similarly to the previous subsection, we have a standard isomorphism of $\mathbb{k}$-algebras

$$
\operatorname{Ext}_{G_{\beta}}^{*}\left(\mathcal{L}_{\beta}^{\bullet}, \mathcal{L}_{\beta}^{\bullet}\right) \cong H_{*}^{G_{\beta}}\left(Z_{\beta}^{\bullet}, \mathbb{k}\right)
$$

which also induces an isomorphism between completions.

Corollary 3.16. We have the following isomorphisms of $\mathbb{k}$-algebras:

$$
\operatorname{Ext}_{G_{\beta}}^{*}\left(\mathcal{L}_{\beta}^{\bullet}, \mathcal{L}_{\beta}^{\bullet}\right)^{\wedge} \cong H_{*}^{G_{\beta}}\left(Z_{\beta}^{\bullet}, \mathbb{k}\right)^{\wedge} \cong \widehat{K}^{G_{\beta}}\left(Z_{\beta}^{\bullet}\right)_{\mathbb{k}} .
$$

\section{Dynkin quiver type quantum affine Schur-Weyl duality}

\subsection{Geometric construction of a bimodule and a Morita equivalence}

We keep the notation in the previous sections. In particular, $\mathbb{k}=\mathbb{Q}(q)$. We fix an element $\beta=\sum_{i \in I} d_{i} \alpha_{i} \in \mathrm{Q}^{+}$and put $\lambda:=\operatorname{cl}\left(\boldsymbol{\lambda}_{\beta}\right) \in \mathrm{P}^{+}$. From the two $G_{\beta}$-equivariant proper morphisms $\pi_{\beta}: \mathfrak{M}_{\beta}^{\bullet} \rightarrow E_{\beta}$ and $\mu_{\beta}: \mathcal{F}_{\beta} \rightarrow E_{\beta}$, we form the fiber product $\mathfrak{M}_{\beta}^{\bullet} \times_{E_{\beta}} \mathcal{F}_{\beta}$. The convolution products make its completed 
$G_{\beta}$-equivariant $K$-group $\widehat{K}^{G_{\beta}}\left(\mathfrak{M}_{\beta}^{\bullet} \times_{E_{\beta}} \mathcal{F}_{\beta}\right)_{\mathbb{k}}$ into a $\left(\widehat{K}^{G_{\beta}}\left(Z_{\beta}^{\bullet}\right)_{\mathbb{k}}, \widehat{K}^{G_{\beta}}\left(\mathcal{Z}_{\beta}\right)_{\mathbb{k}}\right)$ bimodule. More precisely, the convolution products give $\mathbb{k}$-algebra homomorphisms

$$
\widehat{K}^{G_{\beta}}\left(Z_{\beta}^{\bullet}\right)_{\mathbb{k}} \rightarrow \operatorname{End}\left(\widehat{K}^{G_{\beta}}\left(\mathfrak{M}_{\beta}^{\bullet} \times_{E_{\beta}} \mathcal{F}_{\beta}\right)_{\mathbb{k}}\right) \leftarrow \widehat{K}^{G_{\beta}}\left(\mathcal{Z}_{\beta}\right)_{\mathbb{k}}^{\text {op }}
$$

whose images commute with each other. In the rest of this subsection, we prove that this bimodule induces a Morita equivalence.

For a moment, we focus on a component $\mathfrak{M}_{\beta}^{\bullet} \times_{E_{\beta}} \mathcal{F}_{\mathbf{i}}$ for a fixed $\mathbf{i} \in I^{\beta}$. Using the isomorphism $\mathcal{B}_{\mathbf{i}} \cong G_{\beta} / B_{\mathbf{i}}$ with $B_{\mathbf{i}}=\operatorname{Stab}_{G_{\beta}}\left(F_{\mathbf{i}}^{\bullet}\right)$, we have

$$
\begin{aligned}
\mathfrak{M}_{\beta}^{\bullet} \times_{E_{\beta}} \mathcal{F}_{\mathbf{i}} & \cong \mathfrak{M}_{\beta}^{\bullet} \times_{E_{\beta}}\left(G_{\beta} \times{ }^{B_{\mathbf{i}}} \operatorname{pr}_{1}^{-1}\left(F_{\mathbf{i}}^{\bullet}\right)\right) \\
& \cong G_{\beta} \times{ }^{B_{\mathbf{i}}}\left(\mathfrak{M}_{\beta}^{\bullet} \times_{E_{\beta}} \operatorname{pr}_{1}^{-1}\left(F_{\mathbf{i}}^{\bullet}\right)\right),
\end{aligned}
$$

where $\operatorname{pr}_{1}$ is the projection $\mathcal{F}_{\mathbf{i}} \ni\left(F^{\bullet}, x\right) \mapsto F^{\bullet} \in \mathcal{B}_{\mathbf{i}}$. We define a 1-parameter subgroup $\rho_{\mathbf{i}}: \mathbb{C}^{\times} \rightarrow H_{\beta}$ by $\rho_{\mathbf{i}}(t) v_{w_{\mathbf{i}}(k)}:=t^{k} v_{w_{\mathbf{i}}(k)}$ for $t \in \mathbb{C}^{\times}$. Note that this depends on the choice of $w_{\mathbf{i}} \in \mathfrak{S}_{d}$ fixed in Subsection 3.3. We observe that

$$
\operatorname{pr}_{1}^{-1}\left(F_{\mathbf{i}}^{\bullet} \cong\left\{x \in E_{\beta} \mid x\left(F_{\mathbf{i}}^{k}\right) \subset F_{\mathbf{i}}^{k}, \forall k\right\}=\left\{x \in E_{\beta} \mid \lim _{t \rightarrow 0} \rho_{\mathbf{i}}(t) x=0\right\} .\right.
$$

Therefore we get

$$
\mathfrak{M}_{\beta}^{\bullet} \times_{E_{\beta}} \operatorname{pr}_{1}^{-1}\left(F_{\mathbf{i}}^{\bullet}\right) \cong\left\{x \in \mathfrak{M}_{\beta}^{\bullet} \mid \lim _{t \rightarrow 0} \rho_{\mathbf{i}}(t) \pi_{\beta}(x)=0\right\} .
$$

Since the morphism $\pi_{\beta}: \mathfrak{M}_{\beta}^{\bullet} \rightarrow E_{\beta}$ is the $T_{\beta}$-fixed part of $\pi: \mathfrak{M}(\lambda) \rightarrow \mathfrak{M}_{0}(\lambda)$, it is natural to consider the following subvariety of $\mathfrak{M}(\lambda)$ :

$$
\widetilde{\mathfrak{Z}}\left(\lambda ; w_{\mathbf{i}}\right):=\left\{x \in \mathfrak{M}(\lambda) \mid \lim _{t \rightarrow 0} \rho_{\mathbf{i}}(t) \pi(x)=0 \in \mathfrak{M}_{0}(\lambda)\right\},
$$

which turns out to be the tensor product variety introduced by Nakajima [18]. Since the subgroups $T_{\beta}$ and $\rho_{\mathbf{i}}\left(\mathbb{C}^{\times}\right)$commute with each other, we have

$$
\mathfrak{M}_{\beta}^{\bullet} \times_{E_{\beta}} \operatorname{pr}_{1}^{-1}\left(F_{\mathbf{i}}^{\bullet}\right) \cong \widetilde{\mathfrak{Z}}\left(\lambda ; w_{\mathbf{i}}\right)^{T_{\beta}} .
$$

Using (4.1), (4.2) and the reduction, we obtain

$$
\begin{aligned}
K^{G_{\beta}}\left(\mathfrak{M}_{\beta}^{\bullet} \times_{E_{\beta}} \mathcal{F}_{\mathbf{i}}\right) & \cong K^{H_{\beta}}\left(\widetilde{\mathfrak{Z}}\left(\lambda ; w_{\mathbf{i}}\right)^{T_{\beta}}\right), \\
H_{*}^{G_{\beta}}\left(\mathfrak{M}_{\beta}^{\bullet} \times_{E_{\beta}} \mathcal{F}_{\mathbf{i}}, \mathbb{k}\right) & \cong H_{*}^{H_{\beta}}\left(\widetilde{\mathfrak{Z}}\left(\lambda ; w_{\mathbf{i}}\right)^{T_{\beta}}, \mathbb{k}\right) .
\end{aligned}
$$

Proposition 4.1. The $G_{\beta}$-equivariant Chern character map gives an isomorphism:

$$
\operatorname{ch}^{G_{\beta}}: \widehat{K}^{G_{\beta}}\left(\mathfrak{M}_{\beta}^{\bullet} \times_{E_{\beta}} \mathcal{F}_{\mathbf{i}}\right)_{\mathbb{k}} \cong H_{*}^{G_{\beta}}\left(\mathfrak{M}_{\beta}^{\bullet} \times_{E_{\beta}} \mathcal{F}_{\mathbf{i}}, \mathbb{k}\right)^{\wedge} .
$$

Proof. Thanks to (4.3) and (4.4), it is enough to show that the $H_{\beta}$-equivariant Chern character map

$$
\operatorname{ch}^{H_{\beta}}: \widehat{K}^{H_{\beta}}\left(\widetilde{\mathfrak{Z}}\left(\lambda ; w_{\mathbf{i}}\right)^{T_{\beta}}\right)_{\mathbb{k}} \rightarrow H_{*}^{H_{\beta}}\left(\widetilde{\mathfrak{Z}}\left(\lambda, w_{\mathbf{i}}\right)^{T_{\beta}}, \mathbb{k}\right)^{\wedge}
$$

is an isomorphism. This latter assertion follows from a $T_{\beta}$-fixed part analogue of [18, Theorem 3.10. (1)]. 
The $G_{\beta}$-equivariant Borel-Moore homology $H_{*}^{G_{\beta}}\left(\mathfrak{M}_{\beta}^{\bullet} \times_{E_{\beta}} \mathcal{F}_{\beta}, \mathbb{k}\right)$ becomes a $\left(H_{*}^{G_{\beta}}\left(Z_{\beta}^{\bullet}, \mathbb{k}\right), H_{*}^{G_{\beta}}\left(\mathcal{Z}_{\beta}, \mathbb{k}\right)\right)$-bimodule by the convolution products, similarly to the case of $K$-groups. On the other hand, the Ext-group $\operatorname{Ext}_{G_{\beta}}^{*}\left(\mathcal{L}_{\beta}^{\bullet}, \mathcal{L}_{\beta}\right)$ becomes a $\left(\operatorname{Ext}_{G_{\beta}}^{*}\left(\mathcal{L}_{\beta}^{\bullet}, \mathcal{L}_{\beta}^{\bullet}\right), \operatorname{Ext}_{G_{\beta}}^{*}\left(\mathcal{L}_{\beta}, \mathcal{L}_{\beta}\right)\right)$-bimodule by the Yoneda products. This bimodule $\operatorname{Ext}_{G_{\beta}}^{*}\left(\mathcal{L}_{\beta}^{\bullet}, \mathcal{L}_{\beta}\right)$ gives a Morita equivalence between $\operatorname{Ext}_{G_{\beta}}^{*}\left(\mathcal{L}_{\beta}^{\bullet}, \mathcal{L}_{\beta}^{\bullet}\right)$ and $\operatorname{Ext}_{G_{\beta}}^{*}\left(\mathcal{L}_{\beta}, \mathcal{L}_{\beta}\right)$ because $\mathcal{I} \mathcal{C}_{\mathbf{m}}$ appears as a non-zero direct summand of both $\mathcal{L}_{\beta}$ and $\mathcal{L}_{\beta}^{\bullet}$ for each $\mathbf{m} \in \operatorname{KP}(\beta)$. Moreover, we have a standard isomorphism

$$
H_{*}^{G_{\beta}}\left(\mathfrak{M}_{\beta}^{\bullet} \times_{E_{\beta}} \mathcal{F}_{\beta}, \mathbb{k}\right) \cong \operatorname{Ext}_{G_{\beta}}^{*}\left(\mathcal{L}_{\beta}^{\bullet}, \mathcal{L}_{\beta}\right)
$$

Theorem 4.2. We have the following commutative diagram:

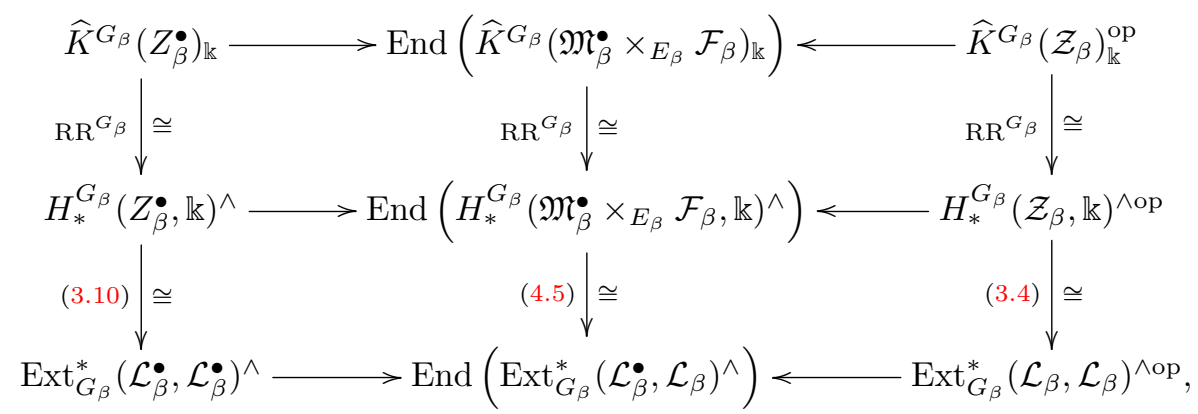

where each row denotes the bimodule structure defined above. In particular, the bimodule $\widehat{K}^{G_{\beta}}\left(\mathfrak{M}_{\beta}^{\bullet} \times_{E_{\beta}} \mathcal{F}_{\beta}\right)_{\mathbb{k}}$ gives a Morita equivalence between two convolution algebras $\widehat{K}^{G_{\beta}}\left(Z_{\beta}^{\bullet}\right)_{\mathbb{k}}$ and $\widehat{K}^{G_{\beta}}\left(\mathcal{Z}_{\beta}\right)_{\mathbb{k}}$.

Proof. The commutativity of the upper half (resp. lower half) of the diagram follows from Proposition 3.3 (resp. an equivariant version of [3, Theorem 8.6.7]).

\subsection{The left action of $U_{q}(L \mathfrak{g})$}

In this subsection, we fix $\mathbf{i}=\left(i_{1}, \ldots, i_{d}\right) \in I^{\beta}$ and investigate the $U_{q}(L \mathfrak{g})$-module structure of the pull-back $\widehat{\Phi}_{\beta}^{*}\left(\widehat{K}^{G_{\beta}}\left(\mathfrak{M}_{\beta}^{\bullet} \times_{E_{\beta}} \mathcal{F}_{\mathbf{i}}\right)_{\mathbb{k}}\right)$.

We use the following notation. For each $i \in I$, we define $\lambda_{i}:=\operatorname{cl}\left(\phi\left(\alpha_{i}\right)\right)=\varpi_{j}$ and $a_{i}:=q^{p}$ if $\phi\left(\alpha_{i}\right)=(j, p) \in \widehat{I}$. Recall from Theorem 3.10 that we have

$$
\operatorname{End}_{U_{q}}\left(\mathbb{W}\left(\lambda_{i}\right)\right) \cong R\left(\mathbb{G}\left(\lambda_{i}\right)\right) \otimes_{A} \mathbb{k}=R\left(G\left(\lambda_{i}\right)\right)_{\mathbb{k}} \cong \mathbb{k}\left[z_{\lambda_{i}}^{ \pm 1}\right],
$$

where $z_{\lambda_{i}}$ denotes the class of the 1-dimensional representation of $G\left(\lambda_{i}\right)=\mathbb{C}^{\times}$ of weight 1 .

We recall some properties of the tensor product variety $\widetilde{\mathfrak{Z}}\left(\lambda ; w_{\mathbf{i}}\right)$. Let

$$
\mathbb{H}_{\beta}:=H_{\beta} \times \mathbb{C}^{\times} \subset G_{\beta} \times \mathbb{C}^{\times}=\mathbb{G}_{\beta} \subset \mathbb{G}(\lambda)
$$

be a maximal torus. By construction, the subvariety $\widetilde{\mathfrak{Z}}\left(\lambda ; w_{\mathbf{i}}\right) \subset \mathfrak{M}(\lambda)$ is stable under the action of $\mathbb{H}_{\beta}$. The convolution product makes the $\mathbb{H}_{\beta}$-equivariant 
$K$-group $K^{\mathbb{H}_{\beta}}\left(\widetilde{\mathfrak{Z}}\left(\lambda ; w_{\mathbf{i}}\right)\right)$ into a left $K^{\mathbb{H}_{\beta}}(Z(\lambda))$-module. Via the composition of the homomorphisms

$$
U_{q}(L \mathfrak{g}) \stackrel{\Phi_{\lambda}}{\longrightarrow} K^{\mathbb{G}(\lambda)}(Z(\lambda)) \otimes_{A} \mathbb{k} \rightarrow K^{\mathbb{H}_{\beta}}(Z(\lambda)) \otimes_{A} \mathbb{k},
$$

where the latter one is the restriction to $\mathbb{H}_{\beta} \subset \mathbb{G}(\lambda)$, we regard the $\mathbb{H}_{\beta^{-}}$ equivariant $K$-group $K^{\mathbb{H}_{\beta}}\left(\widetilde{\mathfrak{Z}}\left(\lambda ; w_{\mathbf{i}}\right)\right) \otimes_{A} \mathbb{k}$ as a $U_{q}(L \mathfrak{g})$-module.

Theorem 4.3 (Nakajima [18]). There is a $U_{q}(L \mathfrak{g})$-module isomorphism

$$
K^{\mathbb{H}_{\beta}}\left(\widetilde{\mathfrak{Z}}\left(\lambda ; w_{\mathbf{i}}\right)\right) \otimes_{A} \mathbb{k} \cong \mathbb{V}^{\otimes \mathbf{i}}:=\mathbb{W}\left(\lambda_{i_{1}}\right) \otimes \cdots \otimes \mathbb{W}\left(\lambda_{i_{d}}\right),
$$

where the action of $R\left(\mathbb{H}_{\beta}\right) \otimes_{A} \mathbb{k}$ on the LHS is translated into the action on the RHS via the isomorphism

$$
\begin{aligned}
R\left(\mathbb{H}_{\beta}\right) \otimes_{A} \mathbb{k} & \stackrel{\cong}{\rightarrow} \mathcal{O}_{\mathbf{i}}:=\mathbb{k}\left[X_{1}^{ \pm 1}, \ldots, X_{d}^{ \pm 1}\right] \subset \operatorname{End}_{U_{q}}\left(\mathbb{V}^{\otimes \mathbf{i}}\right) ; \\
{\left[\mathbb{C} v_{w_{\mathbf{i}}(k)}\right] } & \mapsto X_{k},
\end{aligned}
$$

where we set $X_{k}:=z_{\lambda_{i_{k}}}$ using the notation in (4.6).

The decomposition (2.2) $\mathbb{G}_{\beta} \cong G_{\beta} \times T_{\beta}$ induces the decomposition $\mathbb{H}_{\beta} \cong$ $H_{\beta} \times T_{\beta}$ of the maximal torus $\mathbb{H}_{\beta}$. Similarly to the case of $\mathbb{G}_{\beta}$-equivariant $K$-groups in Subsection 3.4, this decomposition yields a natural isomorphism

$$
K^{\mathbb{H}_{\beta}}(X) \otimes_{A} \mathbb{k} \cong K^{H_{\beta}}(X)_{\mathbb{k}}
$$

for any $\mathbb{H}_{\beta}$-variety $X$ with a trivial $T_{\beta}$-action. When $X=\mathrm{pt}$, we have the following commutative diagram:

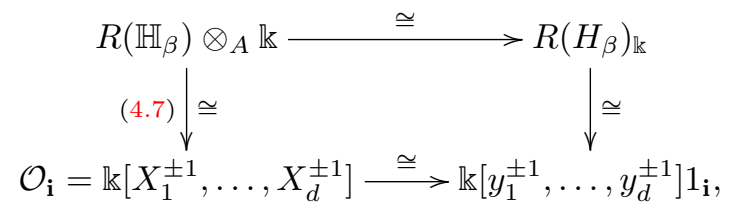

where the bottom horizontal arrow sends the element $a_{i_{k}}^{-1} X_{k}$ to $y_{k} 1_{\mathbf{i}}$ for $1 \leq$ $k \leq d$. Under this isomorphism, the maximal ideal $\mathfrak{r}_{\beta}^{\prime} \subset R\left(\mathbb{H}_{\beta}\right) \otimes_{A} \mathbb{k}$ defined as the kernel of the restriction $R\left(\mathbb{H}_{\beta}\right) \otimes_{A} \mathbb{k} \rightarrow R\left(T_{\beta}\right) \otimes_{A} \mathbb{k}=\mathbb{k}$ corresponds to the augmentation ideal of $R\left(H_{\beta}\right)_{\mathbb{k}}$. Therefore we have a natural isomorphism

$$
\left[K^{\mathbb{H}_{\beta}}(X) \otimes_{A} \mathbb{k}\right]_{\mathfrak{r}_{\beta}^{\prime}}^{\wedge} \cong \widehat{K}^{H_{\beta}}(X)_{\mathbb{k}},
$$

where $[-]_{\mathfrak{r}_{\beta}^{\prime}}^{\wedge}$ denotes the $\mathfrak{r}_{\beta}^{\prime}$-adic completion. In particular, completing the diagram (4.8), we get

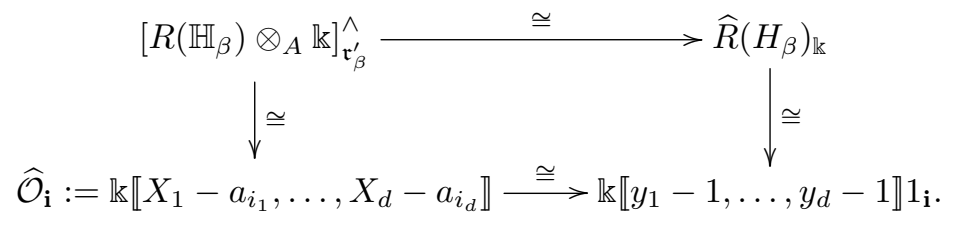


Theorem 4.4. We have the following isomorphism of $U_{q}(L \mathfrak{g})$-modules:

$$
\widehat{\Phi}_{\beta}^{*}\left(\widehat{K}^{G_{\beta}}\left(\mathfrak{M}_{\beta}^{\bullet} \times_{E_{\beta}} \mathcal{F}_{\mathbf{i}}\right)_{\mathbb{k}}\right) \cong \widehat{V}^{\otimes \mathbf{i}}:=\mathbb{V}^{\otimes \mathbf{i}} \otimes_{\mathcal{O}_{\mathbf{i}}} \widehat{\mathcal{O}}_{\mathbf{i}}
$$

Proof. Actually, there is the following isomorphism:

$$
\begin{aligned}
\widehat{V}^{\otimes \mathbf{i}} & \cong\left[K^{\mathbb{H}_{\beta}}\left(\widetilde{\mathfrak{Z}}\left(\lambda ; w_{\mathbf{i}}\right)\right) \otimes_{A} \mathbb{k}\right]_{\mathfrak{r}_{\beta}^{\prime}}^{\wedge} & & (\text { Theorem 4.3) } \\
& \cong\left[K^{\mathbb{H}_{\beta}}\left(\widetilde{\mathfrak{Z}}\left(\lambda ; w_{\mathbf{i}}\right)^{T_{\beta}}\right) \otimes_{A} \mathbb{k}\right]_{\mathfrak{r}_{\beta}^{\prime}}^{\wedge} & & \text { (localization theorem) } \\
& \cong \widehat{K}^{H_{\beta}}\left(\widetilde{\mathfrak{Z}}\left(\lambda ; w_{\mathbf{i}}\right)^{T_{\beta}}\right)_{\mathbb{k}} & & (\text { isomorphism (4.9)) } \\
& \cong \widehat{K}^{G_{\beta}}\left(\mathfrak{M}_{\beta}^{\bullet} \times_{E_{\beta}} \mathcal{F}_{\mathbf{i}}\right)_{\mathbb{k}} . & & \text { (isomorphism (4.3)) }
\end{aligned}
$$

We need to show that this is a $U_{q}(L \mathfrak{g})$-homomorphism. By construction, the following diagram of $\mathbb{k}$-algebras commutes:

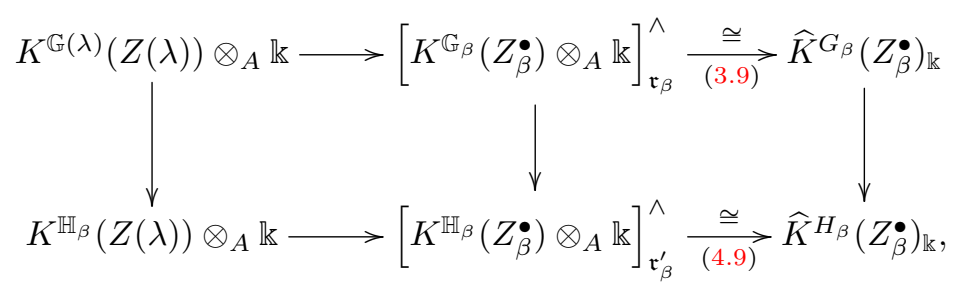

where the vertical arrows denote the restrictions to the maximal tori. Moreover, by using an $H_{\beta}$-equivariant version of [17, Proposition 8.2.3], we can see that the following diagram also commutes:

$$
\begin{gathered}
K^{G_{\beta}}\left(Z_{\beta}^{\bullet}\right)_{\mathbb{k}} \otimes K^{G_{\beta}}\left(\mathfrak{M}_{\beta}^{\bullet} \times_{E_{\beta}} \mathcal{F}_{\mathbf{i}}\right)_{\mathbb{k}} \stackrel{*}{\longrightarrow} K^{G_{\beta}}\left(\mathfrak{M}_{\beta}^{\bullet} \times_{E_{\beta}} \mathcal{F}_{\mathbf{i}}\right)_{\mathbb{k}} \\
\left(\text { restriction to } H_{\beta}\right) \otimes(4.3) \downarrow \\
K^{H_{\beta}}\left(Z_{\beta}^{\bullet}\right)_{\mathbb{k}} \otimes K^{H_{\beta}}\left(\widetilde{\mathfrak{Z}}\left(\lambda ; w_{\mathbf{i}}\right)^{T_{\beta}}\right)_{\mathbb{k}} \stackrel{*}{\longrightarrow} K^{H_{\beta}\left(\widetilde{\mathfrak{Z}}\left(\lambda ; w_{\mathbf{i}}\right)^{T_{\beta}}\right)_{\mathbb{k}},}
\end{gathered}
$$

where the horizontal arrows denote the convolution products. From these commutative diagrams, combined with the definition of $\widehat{\Phi}_{\beta}$ and Theorem 4.3, we obtain the conclusion.

\subsection{The right action of $\widehat{H}_{Q}(\beta)$}

Summarizing the discussion so far, we have obtained a $\left(U_{q}(L \mathfrak{g}), \widehat{H}_{Q}(\beta)\right)$-bimodule structure on the left $U_{q}(L \mathfrak{g})$-module

$$
\widehat{V}^{\otimes \beta}:=\bigoplus_{\mathbf{i} \in I^{\beta}} \widehat{V}^{\otimes \mathbf{i}}
$$


such that the following diagram commutes:

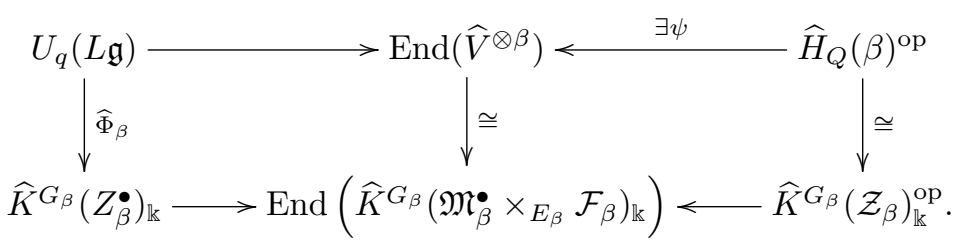

In this subsection, we describe the right action $\psi: \widehat{H}_{Q}(\beta) \rightarrow \operatorname{End}_{U_{q}}\left(\widehat{V}^{\otimes \beta}\right)^{\text {op }}$ of the quiver Hecke algebra $\widehat{H}_{Q}(\beta)$ on the space $\widehat{V}^{\otimes \beta}$.

For each $\mathbf{i}=\left(i_{1}, \ldots, i_{d}\right) \in I^{\beta}$, we set

$$
v_{\mathbf{i}}:=\left(w_{\lambda_{i_{1}}} \otimes \cdots \otimes w_{\lambda_{i_{d}}}\right) \otimes 1 \in \widehat{V}^{\otimes \mathbf{i}}=\left(\mathbb{W}\left(\lambda_{i_{1}}\right) \otimes \cdots \otimes \mathbb{W}\left(\lambda_{i_{d}}\right)\right) \otimes_{\mathcal{O}_{\mathbf{i}}} \widehat{\mathcal{O}}_{\mathbf{i}}
$$

Proposition 4.5. The highest weight space $\bigoplus_{\mathbf{i} \in I^{\beta}} \widehat{\mathcal{O}}_{\mathbf{i}} v_{\mathbf{i}} \subset \widehat{V}^{\otimes \beta}$ of weight $\lambda$ is stable under the right action of $\widehat{H}_{Q}(\beta)$. Moreover it is isomorphic to the completed polynomial representation $\widehat{P}_{\beta}$ defined in (3.1).

Proof. Note that the connected component of the graded quiver variety $\mathfrak{M}_{\beta}^{\bullet}=$ $\mathfrak{M}(\lambda)^{T_{\beta}}$ corresponding to the highest weight space is $\mathfrak{M}(0, \lambda)^{T_{\beta}}=\mathrm{pt}$ and hence $\mathfrak{M}(0, \lambda)^{T_{\beta}} \times_{E_{\beta}} \mathcal{F}_{\beta}=\mathcal{B}_{\beta}$. Therefore we have

$$
\bigoplus_{\mathbf{i} \in I^{\beta}} \widehat{\mathcal{O}}_{\mathbf{i}} v_{\mathbf{i}} \cong \widehat{K}^{G_{\beta}}\left(\mathfrak{M}(0, \lambda)^{T_{\beta}} \times_{E_{\beta}} \mathcal{F}_{\beta}\right)_{\mathbb{k}} \cong \widehat{K}^{G_{\beta}}\left(\mathcal{B}_{\beta}\right)_{\mathbb{k}} \cong \widehat{P}_{\beta}
$$

as $\widehat{H}_{Q}(\beta)$-module, where the last isomorphism comes from (3.3) and (3.7).

Henceforth, we normalize the isomorphism $\widehat{K}^{G_{\beta}}\left(\mathfrak{M}_{\beta}^{\bullet} \times_{E_{\beta}} \mathcal{F}_{\mathbf{i}}\right)_{\mathbb{k}} \cong \widehat{V}^{\otimes \mathbf{i}}$ of $U_{q}(L \mathfrak{g})$-modules in Theorem 4.4 by multiplying the element of $\widehat{\mathcal{O}}_{\mathbf{i}}$ corresponding to the ratio $C_{\mathbf{i}}^{-1}$ of Todd classes defined in (3.6) for each $\mathbf{i} \in I^{\beta}$ so that the isomorphism

$$
\bigoplus_{\mathbf{i} \in I^{\beta}} \widehat{\mathcal{O}}_{\mathbf{i}} v_{\mathbf{i}}=\bigoplus_{\mathbf{i} \in I^{\beta}} \mathbb{k} \llbracket X_{1}-a_{i_{1}}, \ldots, X_{d}-a_{i_{d}} \rrbracket v_{\mathbf{i}} \stackrel{\cong}{\rightarrow} \widehat{P}_{\beta}=\bigoplus_{\mathbf{i} \in I^{\beta}} \mathbb{k} \llbracket x_{1}, \ldots, x_{d} \rrbracket 1_{\mathbf{i}}
$$

in Proposition 4.5 above sends the element $v_{\mathbf{i}}$ to $1_{\mathbf{i}}$.

Now we recall the normalized $R$-matrices. For any pair $\left(i_{1}, i_{2}\right) \in I^{2}$, we simplify $z_{k}:=z_{\lambda_{i_{k}}}$ for $k=1,2$. Then it is known (see e.g. [14, Section 8]) that there is a unique $\left(U_{q} \otimes \mathbb{k}\left[z_{1}^{ \pm 1}, z_{2}^{ \pm 1}\right]\right)$-homomorphism, called the normalized $R$-matrix

$$
R_{i_{1}, i_{2}}^{\text {norm }}: \mathbb{W}\left(\lambda_{i_{1}}\right) \otimes \mathbb{W}\left(\lambda_{i_{2}}\right) \rightarrow \mathbb{k}\left(z_{2} / z_{1}\right) \otimes_{\mathbb{k}\left[\left(z_{2} / z_{1}\right)^{ \pm 1}\right]}\left(\mathbb{W}\left(\lambda_{i_{2}}\right) \otimes \mathbb{W}\left(\lambda_{i_{1}}\right)\right),
$$

such that $R_{i_{1}, i_{2}}^{\text {norm }}\left(w_{\lambda_{i_{1}}} \otimes w_{\lambda_{i_{2}}}\right)=w_{\lambda_{i_{2}}} \otimes w_{\lambda_{i_{1}}}$. The denominator of the normalized $R$-matrix $R_{i_{1}, i_{2}}^{\text {norm }}$ is defined as the monic polynomial $d_{i_{1}, i_{2}}(u) \in \mathbb{k}[u]$ of the smallest degree among polynomials satisfying

$$
\operatorname{Im} R_{i_{1}, i_{2}}^{\text {norm }} \subset d_{i_{1}, i_{2}}\left(z_{2} / z_{1}\right)^{-1} \otimes\left(\mathbb{W}\left(\lambda_{i_{2}}\right) \otimes \mathbb{W}\left(\lambda_{i_{1}}\right)\right) .
$$


By [14, Proposition 9.3], we have

$$
d_{i_{1}, i_{2}}(1) \neq 0 .
$$

Let $\mathbb{K}_{\mathbf{i}}$ be the fraction field of the ring $\widehat{\mathcal{O}}_{\mathbf{i}}$ for each $\mathbf{i} \in I^{\beta}$. It is known that the $U_{q} \otimes \mathbb{K}_{\mathbf{i}}$-module

$$
\widehat{V}_{\mathbb{K}}^{\otimes \mathbf{i}}:=\mathbb{V}^{\otimes \mathbf{i}} \otimes_{\mathcal{O}_{\mathbf{i}}} \mathbb{K}_{\mathbf{i}}=\widehat{V}^{\otimes \mathbf{i}} \otimes_{\widehat{\mathcal{O}}_{\mathbf{i}}} \mathbb{K}_{\mathbf{i}}
$$

is irreducible (see e.g. [14, Proposition 9.5]). For each $w \in \mathfrak{S}_{d}$, the $\mathbb{k}$-algebra isomorphism

$$
\varphi_{w}: \widehat{\mathcal{O}}_{\mathbf{i}} \cong \widehat{\mathcal{O}}_{\mathbf{i} \cdot w} ; \quad f\left(X_{1}, \ldots, X_{d}\right) \mapsto f^{w}\left(X_{1}, \ldots, X_{d}\right):=f\left(X_{w(1)}, \ldots, X_{w(d)}\right)
$$

induces an isomorphism $\mathbb{K}_{\mathbf{i}} \cong \mathbb{K}_{\mathbf{i} \cdot w}$ of the fraction fields, which we denote by the same symbol $\varphi_{w}$. The pull-back $\varphi_{w}^{*} \widehat{V}_{\mathbb{K}}^{\otimes \mathbf{i} \cdot w}$ is an irreducible $U_{q} \otimes \mathbb{K}_{\mathbf{i}}$-module.

For each $\mathbf{i} \in I^{\beta}$ and $1 \leq k<d$, we define the following non-zero $U_{q} \otimes \mathbb{K}_{\mathbf{i}^{-}}$ homomorphism

$$
R_{k}^{\mathbf{i}}:=\left(1^{\otimes(k-1)} \otimes R_{i_{k}, i_{k+1}}^{\text {norm }} \otimes 1^{\otimes(d-k-1)}\right) \otimes \varphi_{s_{k}}: \widehat{V}_{\mathbb{K}}^{\otimes \mathbf{i}} \rightarrow \varphi_{s_{k}}^{*} \widehat{V}_{\mathbb{K}}^{\otimes \mathbf{i} \cdot s_{k}} .
$$

By the irreducibility, this is an isomorphism and we have

$$
\operatorname{Hom}_{U_{q} \otimes \mathbb{K}_{\mathbf{i}}}\left(\widehat{V}_{\mathbb{K}}^{\otimes \mathbf{i}}, \varphi_{s_{k}}^{*} \widehat{V}_{\mathbb{K}}^{\otimes \mathbf{i} \cdot s_{k}}\right)=\mathbb{K}_{\mathbf{i}} \cdot R_{k}^{\mathbf{i}} .
$$

Let $\widehat{V}_{\mathbb{K}}^{\otimes \beta}:=\bigoplus_{\mathbf{i} \in I^{\beta}} \widehat{V}_{\mathbb{K}}^{\otimes \mathbf{i}}$. We regard $\widehat{V}^{\otimes \beta} \subset \widehat{V}_{\mathbb{K}}^{\otimes \beta}$ naturally.

Theorem 4.6. The right action of the quiver Hecke algebra $\widehat{H}_{Q}(\beta)$ on the space $\widehat{V}^{\otimes \beta}$ is given by the following formulas:

$$
\begin{aligned}
v \cdot e\left(\mathbf{i}^{\prime}\right) & =\delta_{\mathbf{i}, \mathbf{i}^{\prime}} v \\
v \cdot x_{k} & =\log \left(a_{i_{k}}^{-1} X_{k}\right) v \\
v \cdot \tau_{k} & = \begin{cases}\left(\log \left(a_{i_{k}}^{-1} X_{k}\right)-\log \left(a_{i_{k+1}}^{-1} X_{k+1}\right)\right)^{-1}\left(R_{k}^{\mathbf{i}}(v)-v\right) & \text { if } i_{k}=i_{k+1}, \\
\left(\log \left(a_{i_{k}}^{-1} X_{k+1}\right)-\log \left(a_{i_{k+1}}^{-1} X_{k}\right)\right) R_{k}^{\mathbf{i}}(v) & \text { if } i_{k} \leftarrow i_{k+1}, \\
R_{k}^{\mathbf{i}}(v) & \text { otherwise, }\end{cases}
\end{aligned}
$$

where $v \in \widehat{V}^{\otimes \mathbf{i}}$ with $\mathbf{i}=\left(i_{1}, \ldots, i_{d}\right) \in I^{\beta}$ and $\log (X):=\sum_{m=1}^{\infty}(-1)^{m+1}(X-$ $1)^{m} / m$.

Proof. The first formula (4.12) is clear from Theorem 3.6 (1) and the construction.

To prove the second formula (4.13), we assume that the vector $v \in \widehat{V}^{\otimes \mathbf{i}}$ corresponds to an element $\zeta \in \widehat{K}^{G_{\beta}}\left(\mathfrak{M}_{\beta}^{\bullet} \times_{E_{\beta}} \mathcal{F}_{\mathbf{i}}\right)_{\mathbb{k}}$ under the isomorphism in Theorem 4.4. By Theorem 3.6 (1), the right action of $e^{x_{k}} \in \widehat{H}_{Q}(\beta)$ on $\widehat{V}^{\otimes \mathbf{i}}$ 
corresponds to the convolution with the class $\Delta_{*}\left[\mathcal{L}_{\mathbf{i}}(k)\right] \in \widehat{K}^{G_{\beta}}\left(\mathcal{F}_{\mathbf{i}} \times_{E_{\beta}} \mathcal{F}_{\mathbf{i}}\right)_{\mathbb{k}}$ from the right, where $\mathcal{L}_{\mathbf{i}}(k)$ is the line bundle on $\mathcal{F}_{\mathbf{i}}$ defined in Subsection 3.3 and $\Delta: \mathcal{F}_{\mathbf{i}} \rightarrow \mathcal{F}_{\mathbf{i}} \times_{E_{\beta}} \mathcal{F}_{\mathbf{i}}$ is the diagonal embedding. By [17, Lemma 8.1.1], we have $\zeta *\left(\Delta_{*}\left[\mathcal{L}_{\mathbf{i}}(k)\right]\right)=\zeta \otimes p_{2}^{*}\left[\mathcal{L}_{\mathbf{i}}(k)\right]$, where $p_{2}: \mathfrak{M}_{\beta}^{\bullet} \times_{E_{\beta}} \mathcal{F}_{\mathbf{i}} \rightarrow \mathcal{F}_{\mathbf{i}}$ is the second projection. The isomorphism (4.3) translates the operation $-\otimes p_{2}^{*}\left[\mathcal{L}_{\mathbf{i}}(k)\right]$ on $K^{G_{\beta}}\left(\mathfrak{M}_{\beta}^{\bullet} \times_{E_{\beta}} \mathcal{F}_{\mathbf{i}}\right)$ into the multiplication of the element $y_{k} 1_{\mathbf{i}} \in R\left(H_{\beta}\right)$ on $K^{H_{\beta}}\left(\widetilde{\mathfrak{Z}}\left(\lambda ; w_{\mathbf{i}}\right)^{T_{\beta}}\right)$. Thus we have $v \cdot e^{x_{k}}=\left(a_{i_{k}}^{-1} X_{k}\right) v$ (see (4.8)).

Let us verify the third formula (4.14). Let $\psi: \widehat{H}_{Q}(\beta) \rightarrow \operatorname{End}_{U_{q}}\left(\widehat{V}^{\otimes \beta}\right)^{\text {op }}$ be the structure morphism. First, we consider the case $i_{k}=i_{k+1}$. From the commutation relation between $e(\mathbf{i}) \tau_{k}$ and $x_{l}$ in $H_{Q}(\beta)$, and the formula (4.13) for $\psi\left(x_{l}\right)$ which we have proved in the previous paragraph, we see that

$$
\left(\mathcal{D} \psi\left(e(\mathbf{i}) \tau_{k}\right)+1\right) f=f^{s_{k}}\left(\mathcal{D} \psi\left(e(\mathbf{i}) \tau_{k}\right)+1\right)
$$

holds in $\operatorname{End}_{U_{q}}\left(\widehat{V}^{\otimes \mathbf{i}}\right)$ for any $f \in \widehat{\mathcal{O}}_{\mathbf{i}}$, where we put $\mathcal{D}:=\log \left(a_{i_{k}}^{-1} X_{k}\right)-\log \left(a_{i_{k+1}}^{-1} X_{k+1}\right)$. In other words, the operator $\mathcal{D} \psi\left(e(\mathbf{i}) \tau_{k}\right)+1$ belongs to $\operatorname{Hom}_{U_{q} \otimes \widehat{\mathcal{O}}_{\mathbf{i}}}\left(\widehat{V}^{\otimes \mathbf{i}}, \varphi_{s_{k}}^{*} \widehat{V}^{\otimes \mathbf{i}}\right)$. Therefore it extends to an operator on the localizations. Namely, we can regard

$$
\mathcal{D} \psi\left(e(\mathbf{i}) \tau_{k}\right)+1 \in \operatorname{Hom}_{U_{q} \otimes \mathbb{K}_{\mathbf{i}}}\left(\widehat{V}_{\mathbb{K}}^{\otimes \mathbf{i}}, \varphi_{s_{k}}^{*} \widehat{V}_{\mathbb{K}}^{\otimes \mathbf{i}}\right) \cong \mathbb{K}_{\mathbf{i}} \cdot R_{k}^{\mathbf{i}},
$$

where the last isomorphism is (4.11). By Proposition 4.5 and the formulas in Theorem 3.5, we see that $\left(\mathcal{D} \psi\left(e(\mathbf{i}) \tau_{k}\right)+1\right) v_{\mathbf{i}}=v_{\mathbf{i}}=R_{k}^{\mathbf{i}}\left(v_{\mathbf{i}}\right)$. Therefore we obtain $\mathcal{D} \psi\left(e(\mathbf{i}) \tau_{k}\right)+1=R_{k}^{\mathbf{i}}$ as an operator on $\widehat{V}^{\otimes \mathbf{i}}$.

The case $i_{k} \neq i_{k+1}$ is easier. In this case, the commutation relation in $H_{Q}(\beta)$ and the formula (4.13) for $\psi\left(x_{l}\right)$ show that the operator $\psi\left(e(\mathbf{i}) \tau_{k}\right)$ already belongs to $\operatorname{End}_{U_{q} \otimes \widehat{\mathcal{O}}_{\mathbf{i}}}\left(\widehat{V}^{\otimes \mathbf{i}}, \varphi_{s_{k}}^{*} \widehat{V}^{\otimes \mathbf{i}}\right)$. Therefore it extends to an element in $\operatorname{Hom}_{U_{q} \otimes \mathbb{K}_{\mathbf{i}}}\left(\widehat{V}_{\mathbb{K}}^{\otimes \mathbf{i}}, \varphi_{s_{k}}^{*} \widehat{V}_{\mathbb{K}}^{\otimes \mathbf{i}}\right)$. Then we proceed just as in the previous paragraph to obtain the desired formula (4.14), taking Proposition 4.5, the formulas in Theorem 3.5 and (4.11) into consideration.

Corollary $4.7\left(=[10]\right.$ Conjecture 4.3.2). For any $i_{1}, i_{2} \in I$, the order of zero of the denominator $d_{i_{1}, i_{2}}(u)$ at the point $u=a_{i_{2}} / a_{i_{1}}$ is at most one.

Proof. Since we know (4.10), we may assume that $i_{1} \neq i_{2}$. We consider a sequence $\mathbf{i}=\left(i_{1}, i_{2}\right) \in I^{\beta}$ with $\beta=\alpha_{i_{1}}+\alpha_{i_{2}}$. When $i_{1} \leftarrow i_{2}$, the formula (4.14) tells us that the operator $\left(\log \left(a_{i_{1}}^{-1} z_{1}\right)-\log \left(a_{i_{2}}^{-1} z_{2}\right)\right) R_{1}^{\mathbf{i}}$ belongs to $\operatorname{Hom}_{U_{q}}\left(\widehat{V}^{\otimes \mathbf{i}}, \widehat{V}^{\otimes \mathbf{i} \cdot s_{1}}\right)$, where we put $z_{k}=z_{\lambda_{i_{k}}}$ for $k=1,2$ as before. Notice that

$$
\log \left(a_{i_{1}}^{-1} z_{1}\right)-\log \left(a_{i_{2}}^{-1} z_{2}\right) \in\left(z_{2} / z_{1}-a_{i_{2}} / a_{i_{1}}\right) \cdot \widehat{\mathcal{O}}_{\mathbf{i}}^{\times} .
$$

Therefore we find that the order of zero of $d_{i_{1}, i_{2}}(u)$ at $u=a_{i_{2}} / a_{i_{1}}$ is at most one. For the other case $i_{k} \nLeftarrow i_{k+1}$, by the formula (4.14), the operator $R_{1}^{\mathbf{i}}$ already belongs to $\operatorname{Hom}_{U_{q}}\left(\widehat{V}^{\otimes \mathbf{i}}, \widehat{V}^{\otimes \mathbf{i} \cdot s_{1}}\right)$. Therefore the order of zero of $d_{i_{1}, i_{2}}(u)$ at $u=a_{i_{2}} / a_{i_{1}}$ is zero. 
Remark 4.8. For each $\mathbf{i} \in I^{\beta}$, we define a topological $\mathbb{k}$-algebra automorphism $\sigma_{\mathbf{i}}$ of $\widehat{\mathcal{O}}_{\mathbf{i}}$ by setting

$$
\sigma_{\mathbf{i}}\left(\log \left(a_{i_{k}}^{-1} X_{k}\right)\right):=a_{i_{k}}^{-1} X_{k}-1
$$

for all $k$. This induces a $U_{q}(L \mathfrak{g})$-module automorphism $\sigma:=\bigoplus_{\mathbf{i} \in I^{\beta}}\left(1 \otimes \sigma_{\mathbf{i}}\right)$ on the module $\widehat{V}^{\otimes \beta}$. If we twist our right $\widehat{H}_{Q}(\beta)$-action by this automorphism $\sigma$ (i.e. we replace the structure map $\psi$ with $\sigma \psi(-) \sigma^{-1}$ ), we get a new right $\widehat{H}_{Q}(\beta)$-action on $\widehat{V}^{\otimes \beta}$ given by the following formulas:

$$
\begin{aligned}
v \cdot e\left(\mathbf{i}^{\prime}\right) & =\delta_{\mathbf{i}, \mathbf{i}^{\prime}} v \\
v \cdot x_{k} & =\left(a_{i_{k}}^{-1} X_{k}-1\right) v \\
v \cdot \tau_{k} & = \begin{cases}\left(a_{i_{k}}^{-1} X_{k}-a_{i_{k+1}}^{-1} X_{k+1}\right)^{-1}\left(R_{k}^{\mathbf{i}}(v)-v\right) & \text { if } i_{k}=i_{k+1}, \\
\left(a_{i_{k}}^{-1} X_{k+1}-a_{i_{k+1}}^{-1} X_{k}\right) R_{k}^{\mathbf{i}}(v) & \text { if } i_{k} \leftarrow i_{k+1}, \\
R_{k}^{\mathbf{i}}(v) & \text { otherwise, }\end{cases}
\end{aligned}
$$

where $v \in \widehat{V}^{\otimes \mathbf{i}}$ with $\mathbf{i}=\left(i_{1}, \ldots, i_{d}\right) \in I^{\beta}$. This new action is same as KangKashiwara-Kim's action in [9], [10].

Theorem 4.9. The formulas (4.12), (4.13) and (4.14) (or the formulas (4.15), (4.16) and (4.17)) define a structure of a $\left(U_{q}(L \mathfrak{g}), \widehat{H}_{Q}(\beta)\right)$-bimodule on the left $U_{q}(L \mathfrak{g})$-module $\widehat{V}^{\otimes \beta}$. The functor $M \mapsto \widehat{V}^{\otimes \beta} \otimes_{\widehat{H}_{Q}(\beta)} M$ gives an equivalence of categories:

$$
\widehat{H}_{Q}(\beta)-\bmod _{\mathrm{fd}} \stackrel{\simeq}{\rightarrow} \mathcal{C}_{Q, \beta} \text {. }
$$

Proof. This follows from the discussions in this subsection, Theorem 3.14 and Theorem 4.2.

\section{References}

[1] V. Chari and A. Pressley. Quantum affine algebras and affine Hecke algebras. Pacific J. Math., 174(2):295-326, 1996.

[2] V. Chari and A. Pressley. Weyl modules for classical and quantum affine algebras. Represent. Theory, 5:191-223, 2001.

[3] N. Chriss and V. Ginzburg. Representation theory and complex geometry. Birkhauser Boston, Inc., Boston, MA, 1997.

[4] D. Edidin and W. Graham. Riemann-Roch for equivariant Chow groups. Duke Math. J., 102(3):567-594, 2000.

[5] R. Fujita. Affine highest weight categories and quantum affine Schur-Weyl duality of Dynkin quiver types. preprint. arXiv:1710.11288.

[6] V. Ginzburg, N. Reshetikhin, and E. Vasserot. Quantum groups and flag varieties. In Mathematical aspects of conformal and topological field theories and quantum groups (South Hadley, MA, 1992), number 175 in Contemp. Math., pages 101-130. Amer. Math. Soc., Providence, RI, 1994. 
[7] D. Happel. Triangulated categories in the representation theory of finitedimensional algebras, volume 119 of London Mathematical Society Lecture Note Series. Cambridge University Press, Cambridge, 1988.

[8] H. Hernandez and B. Leclerc. Quantum Grothendieck rings and derived Hall algebras. J. Reine Angew. Math., 701:77-126, 2015.

[9] S.-J. Kang, M. Kashiwara, and M. Kim. Symmetric quiver Hecke algebras and R-matrices of quantum affine algebras. Invent. Math., 211(2):591-685, 2018.

[10] S.-J. Kang, M. Kashiwara, and M. Kim. Symmetric quiver Hecke algebras and R-matrices of quantum affine algebras, II. Duke Math. J., 164(8):1549$1602,2015$.

[11] S.-J. Kang, M. Kashiwara, M. Kim, and S.-j. Oh. Symmetric quiver Hecke algebras and R-matrices of quantum affine algebras, III. Proc. Lond. Math. Soc. (3),111(2):420-444, 2015.

[12] S.-J. Kang, M. Kashiwara, M. Kim, and S.-j. Oh. Symmetric quiver Hecke algebras and R-matrices of quantum affine algebras, IV. Selecta Math. (N.S.), 22(4):1987-2015, 2016.

[13] M. Kashiwara. Crystal bases of modified quantized enveloping algebra. Duke Math. J., 73(2):383-413, 1994.

[14] M. Kashiwara. On level-zero representations of quantized affine algebras. Duke Math. J., 112(1):117-175, 2002.

[15] S. Kato. Poincare-Birkhoff-Witt bases and Khovanov-Lauda-Rouquier algebras. Duke Math. J., 163(3):619-663, 2014.

[16] M. Khovanov and A. Lauda. A diagrammatic approach to categorification of quantum groups. I. Represent. Theory, 13:309-347, 2009.

[17] H. Nakajima. Quiver varieties and finite-dimensional representations of quantum affine algebras. J. Amer. Math. Soc., 14(1):145-238, 2000.

[18] H. Nakajima. Quiver varieties and tensor products. Invent. Math., 146(2):399-449, 2001.

[19] S.-j. Oh and T. Scrimshaw. Categorical relations between Langlands dual quantum affine algebras: Exceptional cases. preprint. arXiv:1802.09253.

[20] R. Rouquier. 2-Kac-Moody algebras. preprint. arXiv:0812.5023.

[21] M. Varagnolo and E. Vasserot. Canonical bases and KLR-algebras. J. Reine Angew. Math., 659:67-100, 2011. 